\title{
Analytical study of distributed buoyancy sections to control lateral thermal buckling
}

\section{of subsea pipelines}

\author{
Zhenkui Wanga ${ }^{\mathrm{a}}$, Yougang Tang ${ }^{\mathrm{a}}$, G.H.M. van der Heijden ${ }^{\mathrm{b}, *}$ \\ ${ }^{a}$ State Key Laboratory of Hydraulic Engineering Simulation and Safety, Tianjin University, Tianjin 300072, China \\ ${ }^{\mathrm{b}}$ Department of Civil, Environmental and Geomatic Engineering, University College London, London WC1E 6BT, UK \\ Corresponding author: G.H.M. van der Heijden, g.heijden@ucl.ac.uk
}

\begin{abstract}
Unburied subsea pipelines operating under high temperature and high pressure (HT/HP) conditions tend to relieve their axial compressive force by forming lateral buckles in an uncontrolled manner. In order to control lateral buckling, a distributed buoyancy section is often employed. In this study, analytical solutions are deduced for lateral buckling of unburied subsea pipelines with a distributed buoyancy section. An energy analysis is employed to investigate the stability of the buckled pipeline. The influence of the length and weight of the distributed buoyancy section on pipeline buckled configurations, typical lateral buckling behaviour and the minimum critical temperature difference is illustrated and analysed. The results are shown to be in good agreement with experimental data in the literature. The effect of imperfections is also discussed and an error analysis is conducted for one of the main assumptions of the proposed analytical method. The results show that increasing the length or decreasing the weight of the distributed buoyancy section can both be used to decrease the minimum critical temperature difference. The maximum compressive stress will decrease with decreasing weight of the distributed buoyancy section. However, the influence of the length of the distributed buoyancy section on the maximum compressive stress is complicated.
\end{abstract}

Keywords: Subsea pipelines; Lateral buckling; Beam-column; Distributed buoyancy method; Buckle initiation technique.

\section{Nomenclature}

$P_{0}$ is the axial compressive force, induced by high temperature and high pressure, in sections of the pipeline where no axial expansion occurs,

$P$ is the axial compressive force within the buckled section,

$P_{a}$ is the axial compressive force at the virtual anchors between two buckles,

$E I$ is the flexural rigidity of the pipeline ( $E$ is Young's modulus, $I$ is the moment of inertia of the cross-section),

$\lambda$ is an equivalent axial compressive force,

$w_{1}, w_{2}, w_{3}$ are the lateral deflections of the buckled pipeline,

$l_{1}$ is the half-length of the primary lobe of the buckled section,

$l_{2}$ is the half-length of the buckled section,

$l_{b}$ is the half-length of the distributed buoyancy section,

$l_{s}$ is the half-length of the feed-in zone,

$x$ is the longitudinal coordinate along the pipeline,

$W_{b}$ is the submerged weight per unit length of the pipeline with distributed buoyancy section,

$W_{p}$ is the submerged weight per unit length of the pipeline without distributed buoyancy section,

$k=W_{b} / W_{p}$ is a weight ratio coefficient,

$f_{\mathrm{b}}$ and $f_{A b}$ are the lateral and axial soil resistance per unit length for pipeline with distributed buoyancy section, respectively,

$f_{1}$ and $f_{A 1}$ are the lateral and axial soil resistance per unit length for pipeline without distributed buoyancy section, respectively,

$\mu_{L}$ is the coefficient of lateral friction between pipeline and seabed,

$\mu_{A}$ is the coefficient of axial friction between pipeline and seabed,

$A$ is the cross-sectional area of the pipeline, 
$D$ is the external diameter of the pipeline,

$t$ is the wall thickness of the pipeline,

$\alpha$ is the coefficient of linear thermal expansion,

$T_{0}$ is the temperature difference between the pipeline and its surroundings,

$\bar{u}_{i}(i=1-3)$ is the axial deformation of the pipeline,

$u_{1}$ is the length of axial thermal expansion within the feed-in region $0<x<l_{s}$ due to high pressure and high temperature,

$u_{2}$ is the geometric shortening, which allows for the additional length introduced by the lateral displacement,

$w_{m}$ is the lateral displacement amplitude along the pipeline,

$M_{m}$ is the maximum bending moment along the pipeline,

$\sigma_{M}$ is the bending stress along the buckled pipeline,

$\sigma_{m}$ is the maximum axial compressive stress along the pipeline,

$A_{1}-A_{12}$ and $B_{1}-B_{12}$ are constant coefficients,

Case $a$ represents the case $l_{b}<l_{1}$,

Case $b$ represents the case $l_{1}<l_{b}<l_{2}$,

$V$ is the total potential energy relating to the buckled pipeline (in the feed-in region $0 \leq x \leq l_{s}$ ),

$V_{1}$ is the bending strain energy,

$V_{2}$ is the energy loss due to lateral soil resistance,

$V_{3}$ is the energy loss due to axial soil resistance,

$V_{4}$ is the axial compressive strain energy due to the axial compressive force,

$V_{i}$ is the total potential energy of the straight pipeline, namely before buckling,

$u_{20}$ is the geometric shortening induced by initial imperfection.

\section{Introduction}

For the exploitation and transportation of energy resources, subsea pipelines are increasingly being required to operate under high-temperature conditions to ease flow and prevent solidification of the wax fraction in deep water, leading to excessive axial compressive force along the pipeline. Long unburied subsea pipelines tend to relieve their axial compressive force by forming lateral buckles. Such lateral deformations are uncontrolled and may lead to undesirable stresses and strains along the pipeline potentially destroying its integrity [1]. Moreover, the locations of lateral buckles are very uncertain due to complicated breakout soil resistance induced by partial embedment $[2,3]$.

An appropriate method is to introduce some man-made facilities to trigger pipeline to buckle laterally at several planned locations in a controlled manner, rather than to allow it to suffer an uncontrolled, large buckle at one location only [4, 5]. At these planned locations, a sufficient number of lateral buckles should be triggered at a sufficiently low axial compressive force, namely low operating temperature difference. Several buckle initiation techniques, reviewed by Sinclair et al. [6], have recently been employed to ensure that regular buckles form along the pipeline, such as snake-lay, vertical upset and local weight reduction through a distributed buoyancy section [7].

Much of the past work on pipeline buckling is based on Hobbs's work [8,9], which itself is based on the very similar work on the buckling of railway tracks. In this work the whole pipeline is divided into three separate zones, a central buckled region and two adjoining straight regions. Based on this approach, Taylor derived an analytical solution to lateral and upheaval buckling for pipelines with initial imperfection [10-12] and analytical solutions for ideal submarine pipelines by considering a deformation-dependent resistance force model $[13,14]$. A consistent theory is also developed for the analysis of vertical buckling for imperfect heated pipelines by Pedersen and Jensen [15].

More recently, Hobbs's method has been adopted by several other studies. Wang and Shi $[16,17]$ investigated the upheaval buckling for ideal straight pipelines and for pipelines with prop imperfection on a plastic soft seabed. Also, analytical solutions were proposed and compared with finite-element simulations for high-order buckling modes of ideal pipelines and subsea pipelines with a single-arch initial imperfection $[18,19]$, which were all based on the classical lateral buckling modes proposed by Hobbs. Karampour and co-workers investigated the interaction between upheaval or lateral buckling and propagation 
buckling of subsea pipelines [20-22]. Zhu [23] and Wang [24] proposed a new approach for determining the lateral buckling behaviour of pipelines under thermal loading without the assumption of lateral configuration. In addition, many finite-element analyses have been performed to investigate lateral and upheaval buckling [25-29]. All these studies focussed on lateral buckling or vertical buckling behaviour rather than on how to control this behaviour.

In recent years, lateral buckling of subsea pipelines with buoyancy sections has been studied by several researchers. In the distributed buoyancy method discrete lengths of typically $60 \mathrm{~m}$ to $200 \mathrm{~m}$ of pipeline are installed with additional buoyancy on them; these lengths are intended as buckle initiation sites. The buoyancy is chosen so that the operational submerged weight is a small fraction (typically 10\% to 15\%) of the submerged weight of the normal pipe. This method also reduces the restraint provided by the soil, reducing loads in operation [6]. Simple analytical solutions were given for triggering lateral buckles by applying buoyancy to the pipeline by Peek and Yun, which are valid for a single-point buoyancy load, two-point buoyancy load and distributed buoyancy load over a specified length [30]. They assumed that the pipeline was uplifted over a certain length within the buckled section. The lateral soil resistance was taken to be zero over this uplifted section and the bifurcation load to initiate lateral buckling was derived. However, in some cases, the pipeline is not fully uplifted off the seabed. Thus, the effect of buoyancy section is partially to reduce lateral soil resistance, which is not reduced to zero. This situation was not considered by Peek and Yun. The single buoyancy load required to trigger lateral buckles along a pipeline was investigated through analytical methods by Shi and Wang [4]. The pipeline was divided into three zones in the horizontal plane: the span zone, in which the pipeline is uplifted by the buoyancy force, and two contacting zones, in which the pipeline contacts the seabed. The lateral soil resistance within the span zone was taken zero, while the lateral soil resistance within the contacting zones was assumed elastic, which is not realistic in practice. Analytical solutions based on the third lateral buckling mode (in Hobbs's classification) for a pipeline with a distributed buoyancy section were derived by Antunes [31], which could be employed in the preliminary design to assess the effect of a buoyancy section. An analytical solution was derived for the lateral buckling of the pipeline with buoyancy section by Li [32] and Wang [33]. Compared to their study, one improvement made in this paper is that the assumption of a constant axial compressive force is only employed in the calculation of the lateral deformation and not in the calculation of axial force balance and axial thermal expansion.

In this paper an analytical solution for the lateral buckling of subsea pipelines with a distributed buoyancy section is derived. The solution is used to study the influence of properties of the buoyancy section on lateral buckling. First, the stability of a typical buckling path is analysed by computing the total energy of the pipeline. Then, the influence of the length and weight of the distributed buoyancy section on pipeline buckled configurations and typical lateral buckling behaviour is presented and analysed. The minimum critical temperature difference, lateral displacement amplitude and maximum axial compressive stress are illustrated and discussed in detail. The effect of imperfections is also analysed. Finally, an error analysis is presented and the analytical model is validated by comparison of its predictions with the experimental data reported in [34]. Good agreement is found.

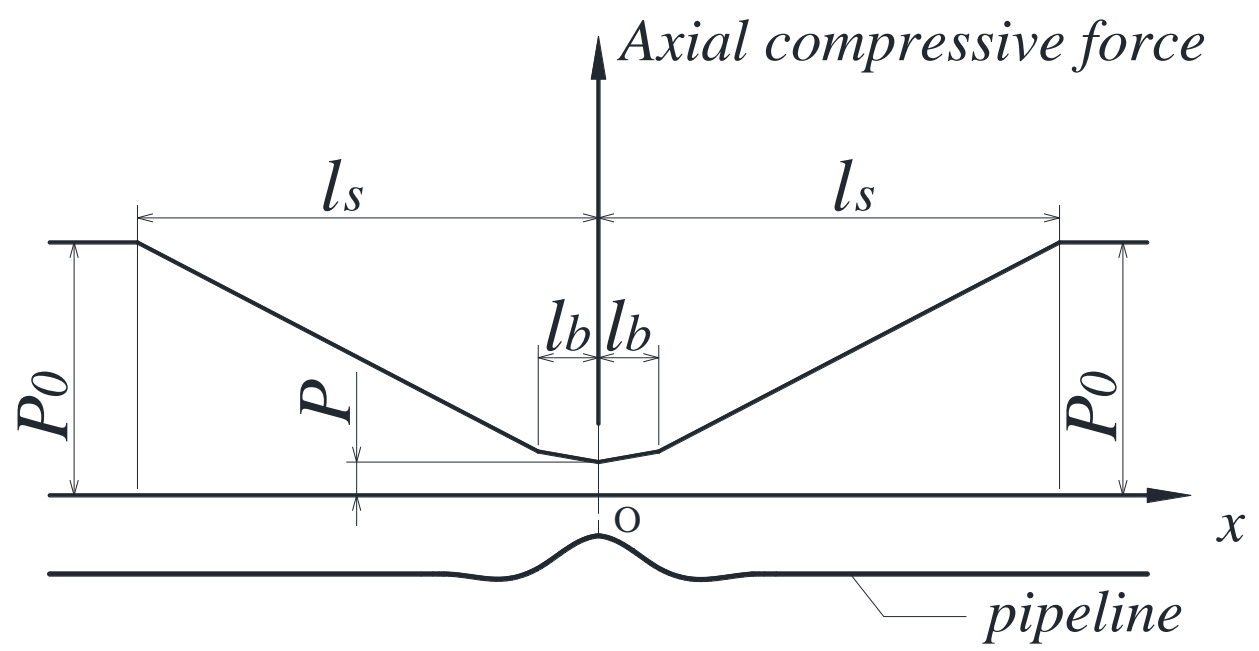

Fig. 1 Axial compressive force distribution. 


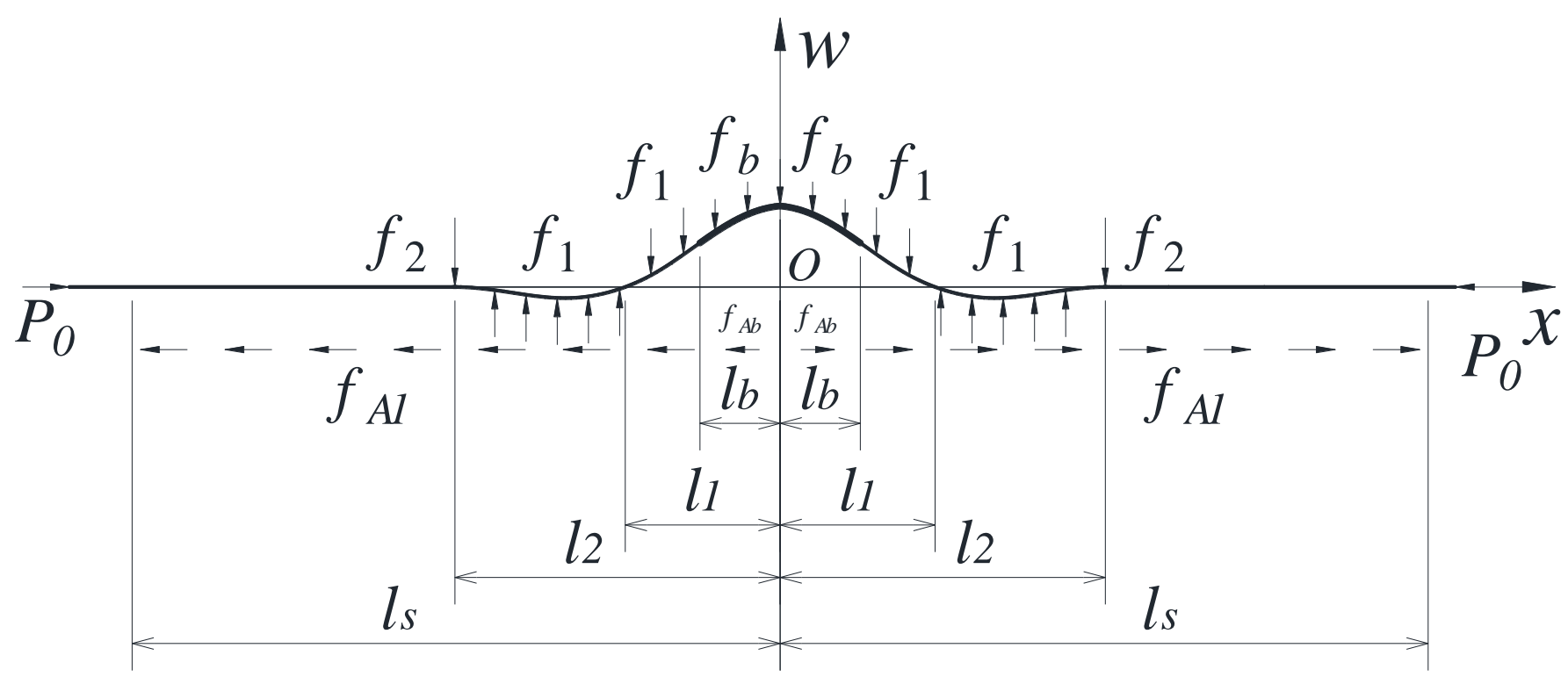

Fig. 2 Configuration and loads distribution of the third lateral buckling mode (case $a$ for $l_{b}<l_{1}$ ). The buoyancy section, of length $2 l_{b}$, is indicated by the thick line.

\section{Analytical solution}

Before buckling, the axial compressive force, $P_{0}$, along the whole pipeline is induced by thermal expansion. In the process of thermal buckling within a pipeline section that is initially immobilised by axial soil resistance against the seabed a small central segment of pipeline will mobilise. As pipe feeds into the buckle the compressive force in the pipe drops, pulling more pipe into the buckle. If the soil resistance for axial movement is constant, say $f_{A}$, then a compressive force will build up in the pipe, increasing linearly with the distance from the centre of the pipeline. At some point this compressive force is sufficient to satisfy the requirement of additional length introduced by the lateral displacement. The end points of this segment are called virtual anchor points. Fig. 1 shows this feed-in region, of length $2 l_{s}$, within the larger immobilised section of the pipeline together with the typical compressive force variation. $l_{s}$ is sometimes called the slip-length. The axial compressive force at the virtual anchor points is $P_{0}$.

The pipeline is more likely to buckle at the locations where the buoyancy is applied due to the following combined advantages. The initiation method of buoyancy works in two ways. First, during lay the section of pipe with buoyancy will be positively buoyant. This means that it tends to form vertical imperfections as it is laid down. This is further exaggerated by the increase in outside diameter and the reduction in vertical load associated with the buoyancy modules. These vertical imperfections, coupled with the effect of hydrodynamic loading, will tend to produce a natural out-of-straightness at the chosen location. Second, because the operational submerged weight is so low, the lateral frictional restraint is reduced. Consequently, the buckle initiation force is also reduced. So an assumption is made that buckling happens at the buoyancy section, symmetric about the centre of this section. Another assumption is that there is no uplift of the pipeline and therefore take the lateral friction coefficient $\mu_{L}$ to be the same for the buoyancy section and the normal sections of the pipeline.

In practice multiple (independent) localised buckles may form in the immobilised pipe section if multiple buoyancy sections are employed. In the following a theory is presented for a single localised buckle that applies to each such buckle individually. Fig. 2 illustrates the configuration and load distribution of the third lateral buckling mode for unburied subsea pipelines with a distributed buoyancy section. $W_{b}$ and $W_{p}$ are the submerged weight per unit length of the buoyancy and normal sections of the pipeline, respectively. Let $W_{b}=k W_{p} ; k$ is a dimensionless ratio. $f_{b}$ and $f_{1}$ are the lateral soil resistance of the buoyancy and normal sections, respectively, and $f_{A b}$ and $f_{A 1}$ are the axial soil resistance of the buoyancy and normal sections, respectively. $l_{b}$ is the half-length of the buoyancy section. $l_{1}$ is the half-length of the primary lobe. $l_{2}$ is the halflength of whole buckled section. In the analytical formulations of this mode presented in this section the pipeline is modelled using linear beam-column theory valid for small deflections. 
With reference to Fig. 1, the axial compressive force distribution $\bar{P}(x)$ can be expressed as

$$
\bar{P}(x)= \begin{cases}P+f_{A b} x & 0 \leq x \leq l_{b} \\ P+f_{A b} l_{b}+f_{A 1}\left(x-l_{b}\right) & l_{b} \leq x \leq l_{s}\end{cases}
$$

The axial soil resistance (a force per unit length) can be written as

$$
f_{A b}=\mu_{A} W_{b}, f_{A 1}=\mu_{A} W_{p}
$$

where $P$ is the axial compressive force at the centre of the buckle. $\mu_{A}$ is the coefficient of axial friction between pipeline and seabed.

Now the assumption is made that the axial compressive force is constant in the buckled region and equal to the force at the centre of the buckle, i.e., $P$. The same approximation was made by Hobbs [9]. The lateral soil resistance is also assumed constant. Two situations are considered in this paper, namely case $a$ for $l_{b}<l_{1}$ and case $b$ for $l_{1}<l_{b}<l_{2}$. Thus, the governing equations that determine the configuration of the buckled pipeline are

For case $a\left(l_{b}<l_{1}\right)$

For case $b\left(l_{1}<l_{b}<l_{2}\right)$

$$
\begin{cases}E I \frac{d^{4} w_{1}}{\mathrm{~d} x^{4}}+P \frac{d^{2} w_{1}}{\mathrm{~d} x^{2}}=-f_{b} & \left(0 \leq x \leq l_{b}\right) \\ E I \frac{d^{4} w_{2}}{\mathrm{~d} x^{4}}+P \frac{d^{2} w_{2}}{\mathrm{~d} x^{2}}=-f_{1} & \left(l_{b} \leq x \leq l_{1}\right) \\ E I \frac{d^{4} w_{3}}{\mathrm{~d} x^{4}}+P \frac{d^{2} w_{3}}{\mathrm{~d} x^{2}}=f_{1} \quad\left(l_{1} \leq x \leq l_{2}\right)\end{cases}
$$

$$
\left\{\begin{array}{c}
E I \frac{d^{4} w_{1}}{\mathrm{~d} x^{4}}+P \frac{d^{2} w_{1}}{\mathrm{~d} x^{2}}=-f_{b} \quad\left(0 \leq x \leq l_{1}\right) \\
E I \frac{d^{4} w_{2}}{\mathrm{~d} x^{4}}+P \frac{d^{2} w_{2}}{\mathrm{~d} x^{2}}=f_{b} \quad\left(l_{1} \leq x \leq l_{b}\right) \\
E I \frac{d^{4} w_{3}}{\mathrm{~d} x^{4}}+P \frac{d^{2} w_{3}}{\mathrm{~d} x^{2}}=f_{1} \quad\left(l_{b} \leq x \leq l_{2}\right)
\end{array}\right.
$$

where $w_{1}, w_{2}, w_{3}$ are the lateral deflections, $E$ is the elastic modulus and $I$ is the moment of inertia of the pipe's crosssection.

The lateral soil resistance (a force per unit length) can be written as

$$
f_{b}=\mu_{L} W_{b}, f_{1}=\mu_{L} W_{p}
$$

where $\mu_{L}$ is lateral friction coefficient.

Let

$$
\lambda^{2}=\frac{P}{E I}
$$

The general solutions of Eq. (3) and Eq. (4) are

For case $a\left(0<l_{b}<l_{1}\right)$

$$
\begin{cases}w_{1}(x)=A_{1} \cos \lambda x+A_{2} \sin \lambda x+A_{3} x+A_{4}-\frac{f_{b} x^{2}}{2 E I \lambda^{2}} & \left(0 \leq x \leq l_{b}\right) \\ w_{2}(x)=A_{5} \cos \lambda x+A_{6} \sin \lambda x+A_{7} x+A_{8}-\frac{f_{1} x^{2}}{2 E I \lambda^{2}} & \left(l_{b} \leq x \leq l_{1}\right) \\ w_{3}(x)=A_{9} \cos \lambda x+A_{10} \sin \lambda x+A_{11} x+A_{12}+\frac{f_{1} x^{2}}{2 E I \lambda^{2}} & \left(l_{1} \leq x \leq l_{2}\right)\end{cases}
$$

For case $b\left(l_{1}<l_{b}<l_{2}\right)$

$$
\begin{cases}w_{1}(x)=B_{1} \cos \lambda x+B_{2} \sin \lambda x+B_{3} x+B_{4}-\frac{f_{b} x^{2}}{2 E I \lambda^{2}} & \left(0 \leq x \leq l_{1}\right) \\ w_{2}(x)=B_{5} \cos \lambda x+B_{6} \sin \lambda x+B_{7} x+B_{8}+\frac{f_{b} x^{2}}{2 E I \lambda^{2}} & \left(l_{1} \leq x \leq l_{b}\right) \\ w_{3}(x)=B_{9} \cos \lambda x+B_{10} \sin \lambda x+B_{11} x+B_{12}+\frac{f_{1} x^{2}}{2 E I \lambda^{2}} & \left(l_{b} \leq x \leq l_{2}\right)\end{cases}
$$

By symmetry, the slope of the deflection and the shear force at $x=0$ must be zero. In addition, the displacement, slope and moment at $x=l_{2}$ must be zero too. So the boundary conditions at $x=0$ and $x=l_{2}$ are 


$$
\left\{\begin{array}{l}
\frac{d w_{1}}{d x}(0)=0 \\
\frac{d^{3} w_{1}}{d x^{3}}(0)=0 \\
w_{3}\left(l_{2}\right)=0 \\
\frac{d w_{3}}{d x}\left(l_{2}\right)=0 \\
\frac{d^{2} w_{3}}{d x^{2}}\left(l_{2}\right)=0
\end{array}\right.
$$

In addition, the displacement, slope, moment and shear force must be continuous at $x=l_{1}$ and $x=l_{b}$, while the displacement at $x=l_{1}$ must be zero. So the following matching conditions at $x=l_{1}$ and $x=l_{b}$ are imposed.

For case $a\left(0<l_{b}<l_{1}\right)$

For case $b\left(l_{1}<l_{b}<l_{2}\right)$

$$
\begin{gathered}
\left\{\begin{array}{c}
w_{2}\left(l_{1}\right)=w_{3}\left(l_{1}\right) \\
\frac{d w_{2}}{d x}\left(l_{1}\right)=\frac{d w_{3}}{d x}\left(l_{1}\right) \\
\frac{d^{2} w_{2}}{d x^{2}}\left(l_{1}\right)=\frac{d^{2} w_{3}}{d x^{2}}\left(l_{1}\right) \\
\frac{d^{3} w_{2}}{d x^{3}}\left(l_{1}\right)=\frac{d^{3} w_{3}}{d x^{3}}\left(l_{1}\right) \\
w_{2}\left(l_{1}\right)=0
\end{array}\right. \\
\left\{\begin{array}{c}
w_{1}\left(l_{\mathrm{b}}\right)=w_{2}\left(l_{\mathrm{b}}\right) \\
\frac{d w_{1}}{d x}\left(l_{\mathrm{b}}\right)=\frac{d w_{2}}{d x}\left(l_{\mathrm{b}}\right) \\
\frac{d^{2} w_{1}}{d x^{2}}\left(l_{\mathrm{b}}\right)=\frac{d^{2} w_{2}}{d x^{2}}\left(l_{\mathrm{b}}\right) \\
\frac{d^{3} w_{1}}{d x^{3}}\left(l_{\mathrm{b}}\right)=\frac{d^{3} w_{2}}{d x^{3}}\left(l_{\mathrm{b}}\right)
\end{array}\right.
\end{gathered}
$$

The overall lateral force balance is

$$
\begin{gathered}
\left\{\begin{array}{c}
w_{1}\left(l_{1}\right)=w_{2}\left(l_{1}\right) \\
\frac{d w_{1}}{d x}\left(l_{1}\right)=\frac{d w_{2}}{d x}\left(l_{1}\right) \\
\frac{d^{2} w_{1}}{d x^{2}}\left(l_{1}\right)=\frac{d^{2} w_{2}}{d x^{2}}\left(l_{1}\right) \\
\frac{d^{3} w_{1}}{d x^{3}}\left(l_{1}\right)=\frac{d^{3} w_{2}}{d x^{3}}\left(l_{1}\right) \\
w_{2}\left(l_{1}\right)=0
\end{array}\right. \\
\left\{\begin{array}{c}
w_{2}\left(l_{\mathrm{b}}\right)=w_{3}\left(l_{\mathrm{b}}\right) \\
\frac{d w_{2}}{d x}\left(l_{\mathrm{b}}\right)=\frac{d w_{3}}{d x}\left(l_{\mathrm{b}}\right) \\
\frac{d^{2} w_{2}}{d x^{2}}\left(l_{\mathrm{b}}\right)=\frac{d^{2} w_{3}}{d x^{2}}\left(l_{\mathrm{b}}\right) \\
\frac{d^{3} w_{2}}{d x^{3}}\left(l_{\mathrm{b}}\right)=\frac{d^{3} w_{3}}{d x^{3}}\left(l_{\mathrm{b}}\right)
\end{array}\right.
\end{gathered}
$$

$$
f_{2}= \begin{cases}f_{1}\left(l_{2}-2 l_{1}+l_{\mathrm{b}}\right)-f_{\mathrm{b}} l_{\mathrm{b}} & \text { for case } a \\ f_{1}\left(l_{2}-l_{\mathrm{b}}\right)+f_{\mathrm{b}}\left(l_{\mathrm{b}}-2 l_{1}\right) & \text { for case } b\end{cases}
$$

for the point force at $x=l_{2}$. This $f_{2}$ is required to prevent a lobe forming in the horizontal plane. (The pipeline is not constrained in the horizontal plane in practice, only resisted by lateral soil resistance, so in general further oscillations or lobes may form. By assuming that these do not form, i.e., that a third mode of lateral buckling is formed, the immobilised part of the pipeline for $x>l_{2}$ is effectively considered to provide a rigid support against which the buckled part of the pipeline pushes. This requires the point force $f_{2}$ at $x=l_{2}$. However, the deformed shape and the buckling path can be predicted accurately by using the assumption of a third mode [24], so this assumption is employed in this paper.)

Axial deformation of the pipeline within the section $l_{2} \leq x \leq l_{\mathrm{s}}$ is governed by the equation

$$
E A \frac{d^{2} \bar{u}_{1}}{d x^{2}}=f_{A 1} \quad\left(l_{2} \leq x \leq l_{\mathrm{s}}\right)
$$

where $A$ is the cross-sectional area of the pipeline. Eq. (15) is solved subject to the slip-length boundary conditions [10] 


$$
\left\{\begin{array}{l}
\bar{u}_{1}\left(l_{\mathrm{s}}\right)=0 \\
\frac{d \bar{u}_{1}}{d x}\left(l_{\mathrm{s}}\right)=0
\end{array}\right.
$$

giving for the axial displacement

$$
\bar{u}_{1}(x)=\frac{f_{A 1}}{2 E A}\left(x-l_{\mathrm{s}}\right)^{2} \quad\left(l_{2} \leq x \leq l_{\mathrm{s}}\right)
$$

Based on Eq. (17), the axial displacement at $x=l_{2}$ is

$$
\bar{u}_{1}\left(l_{2}\right)=\frac{f_{A 1}}{2 E A}\left(l_{2}-l_{\mathrm{s}}\right)^{2}
$$

Axial deformation of the pipeline within the buckled section $0 \leq x \leq l_{2}$ is governed by the equation

$$
\left\{\begin{array}{l}
E A \frac{d^{2} \bar{u}_{2}}{d x^{2}}=f_{A b}\left(0 \leq x \leq l_{\mathrm{b}}\right) \\
E A \frac{d^{2} \bar{u}_{3}}{d x^{2}}=f_{A 1}\left(l_{\mathrm{b}} \leq x \leq l_{2}\right)
\end{array}\right.
$$

with two boundary conditions

$$
\left\{\begin{array}{c}
\bar{u}_{2}(0)=0 \\
\bar{u}_{3}\left(l_{2}\right)=\bar{u}_{1}\left(l_{2}\right)
\end{array}\right.
$$

and two matching conditions at $l_{\mathrm{b}}$

$$
\left\{\begin{aligned}
\bar{u}_{2}\left(l_{\mathrm{b}}\right) & =\bar{u}_{3}\left(l_{\mathrm{b}}\right) \\
\frac{d \bar{u}_{2}}{d x}\left(l_{\mathrm{b}}\right) & =\frac{d \bar{u}_{3}}{d x}\left(l_{\mathrm{b}}\right)
\end{aligned}\right.
$$

The general solutions of Eq. (19) are obtained as follows:

$$
\begin{cases}\bar{u}_{2}(x)=\frac{\left(-f_{A 1}\left(l_{2}-l_{\mathrm{b}}\right)^{2}+f_{A b} l_{\mathrm{b}}\left(-2 l_{2}+l_{\mathrm{b}}\right)+f_{A 1}\left(l_{2}-l_{\mathrm{S}}\right)^{2}\right) x}{2 E A l_{2}}+\frac{f_{A b} x^{2}}{2 E A} & \left(0 \leq x \leq l_{\mathrm{b}}\right) \\ \bar{u}_{3}(x)=\frac{\left(f_{A 1}-f_{A b}\right) l_{\mathrm{b}}{ }^{2}}{2 E A}+\frac{\left(f_{A b} l_{\mathrm{b}}{ }^{2}-f_{A 1}\left(l_{2}{ }^{2}+l_{\mathrm{b}}{ }^{2}\right)+f_{A 1}\left(l_{2}-l_{\mathrm{S}}\right)^{2}\right) x}{2 E A l_{2}}+\frac{f_{A 1} x^{2}}{2 E A} & \left(l_{\mathrm{b}} \leq x \leq l_{2}\right)\end{cases}
$$

$\bar{u}_{1}(x), \bar{u}_{2}(x)$ and $\bar{u}_{3}(x)$ will be used later when computing the total potential energy of a pipeline buckling solution. The axial force balance is also noted:

$$
P_{0}=P+f_{A b} l_{\mathrm{b}}+f_{A 1}\left(l_{\mathrm{s}}-l_{\mathrm{b}}\right)
$$

Now the compatibility between axial and lateral deformation in the feed-in region $0 \leq x \leq l_{s}$ is used to derive a relationship between the axial compressive force $P$ at the centre of the pipeline and the temperature difference $T_{0}$. Compatibility can be expressed as

$$
u_{1}=u_{2}
$$

where $u_{1}$ is the length of axial thermal expansion within the feed-in region $0<x<l_{s}$ due to high pressure and high temperature. $u_{2}$ is the geometric shortening, which allows for the additional length introduced by the lateral displacement. Eq. (24) simply states that, since there are virtual anchor points at distance $l_{\mathrm{s}}$ from the centre of the pipe, the extra length of pipe in the buckle must come from axial expansion of the mobilised section of pipeline.

The length of axial thermal expansion $u_{1}$ can be obtained by

$$
u_{1}=\int_{0}^{l_{s}} \frac{\Delta \bar{P}(x)}{E A} d x
$$

where $\Delta \bar{P}(x)$ is the amount of decrease of axial compressive force along the pipeline after the pipeline buckles, given by

$$
\Delta \bar{P}(x)= \begin{cases}P_{0}-P-f_{A b} x & 0<x<l_{\mathrm{b}} \\ P_{0}-P-f_{A b} l_{\mathrm{b}}-f_{A 1}\left(x-l_{\mathrm{b}}\right) & l_{\mathrm{b}}<x<l_{s}\end{cases}
$$

Thus, $u_{1}$ is expressed as

$$
u_{1}=\frac{1}{2 E A}\left(f_{A 1}\left(l_{s}^{2}-l_{b}^{2}\right)+f_{A b} l_{b}^{2}\right)
$$

and by combining Eq. (24) and Eq. (27) $l_{s}$ can be written as 


$$
l_{S}=\sqrt{\frac{\left(f_{A 1}-f_{A b}\right) l_{b}^{2}+2 A E u_{2}}{f_{A 1}}}
$$

Meanwhile, $u_{2}$ can be obtained as

$$
u_{2}= \begin{cases}\frac{1}{2} \int_{0}^{l_{b}}\left(\frac{d w_{1}}{d x}\right)^{2} d x+\frac{1}{2} \int_{l_{b}}^{l_{1}}\left(\frac{d w_{2}}{d x}\right)^{2} d x+\frac{1}{2} \int_{l_{1}}^{l_{2}}\left(\frac{d w_{3}}{d x}\right)^{2} d x & \text { for case } a \\ \frac{1}{2} \int_{0}^{l_{1}}\left(\frac{d w_{1}}{d x}\right)^{2} d x+\frac{1}{2} \int_{l_{1}}^{l_{b}}\left(\frac{d w_{2}}{d x}\right)^{2} d x+\frac{1}{2} \int_{l_{b}}^{l_{2}}\left(\frac{d w_{3}}{d x}\right)^{2} d x & \text { for case } b\end{cases}
$$

Combining Eq. (23) and Eq. (28), finally the following relationship is obtained:

$$
P_{0}=P+f_{A b} l_{b}+f_{A 1}\left(-l_{b}+\sqrt{\frac{\left(f_{A 1}-f_{A b}\right) l_{b}^{2}+2 A E u_{2}}{f_{A 1}}}\right)
$$

Within the range of linear elastic response this compressive force $P_{0}$ can be written as

$$
P_{0}=E A \alpha T_{0}
$$

where $\alpha$ is the coefficient of linear thermal expansion. $T_{0}$ is the total temperature difference, which is composed of the initial temperature difference and the equivalent temperature difference generated by internal pressure [35].

Given $T_{0}$, Eq. (30) (with Eq. (29) and Eq. (31) inserted) is solved in conjunction with Eq. (9) and Eq. (10) and Eq. (11) (for case $a$ )) or Eq. (12) and Eq. (13) (for case $b$ ) to obtain $P, l_{1}, l_{2}$ and the coefficients $A_{1}-A_{12}$ (case $a$ ) or $B_{1}-B_{12}$ (case $b$ ) (i.e., 15 equations for 15 unknowns) and hence the lateral deflections $w_{1}, w_{2}$ and $w_{3}$. The solutions of $A_{1}-A_{12}$ and $B_{1}-B_{12}$ in terms of $P, l_{1}, l_{2}$ and the physical parameters of the problem are given in the appendix.

The bending moment $M_{m}$ along the buckled pipeline can be computed as

$$
M_{m}=E I \frac{d^{2} w}{d x^{2}}
$$

and the corresponding bending stress $\sigma_{M}$ along the buckled pipeline is given by

$$
\sigma_{M}=\frac{M_{m} D}{2 I}
$$

The maximum stress $\sigma_{m}$ along the pipeline induced by axial compressive force $P$ and bending moment $M_{m}$ can be obtained through the following expression:

$$
\sigma_{m}=\frac{P}{A}+\frac{M_{m} D}{2 I}
$$

\section{Results}

\subsection{Energy analysis}

The typical relationship between lateral buckling amplitude $w_{m}$ (in this study always $w_{m}=w(0)$ ) and total temperature difference $T_{0}$ for a typical solution with distributed buoyancy section is shown in Fig. $3 . k=1$ in Fig. 3 represents the case for pipeline without distributed buoyancy section. The significant point $m$ along the post-buckling path corresponds to the minimum critical temperature difference $T_{m} . T_{m}=20.49{ }^{\circ} \mathrm{C}$ for this case (discussed below). For $T_{0}>T_{m}$ two solution branches exist, which will be referred to as $m-b$ and $m-c$, as shown in Fig. 3. The energies of these two branches are compared to assess the stability of these two branches.

The total potential energy relating to the buckled pipeline (in the feed-in region $0 \leq x \leq l_{s}$ ) is given by

$$
V=V_{1}+V_{2}+V_{3}+V_{4}
$$

The bending strain energy $V_{1}$ can be expressed as

$$
V_{1}=\left\{\begin{array}{l}
\frac{1}{2} E I \int_{0}^{l_{\mathrm{b}}}\left(\frac{d^{2} w_{1}}{d x^{2}}\right)^{2} d x+\frac{1}{2} E I \int_{l_{\mathrm{b}}}^{l_{1}}\left(\frac{d^{2} w_{2}}{d x^{2}}\right)^{2} d x+\frac{1}{2} E I \int_{l_{1}}^{l_{2}}\left(\frac{d^{2} w_{3}}{d x^{2}}\right)^{2} d x \text { for case } a \\
\frac{1}{2} E I \int_{0}^{l_{1}}\left(\frac{d^{2} w_{1}}{d x^{2}}\right)^{2} d x+\frac{1}{2} E I \int_{l_{1}}^{l_{\mathrm{b}}}\left(\frac{d^{2} w_{2}}{d x^{2}}\right)^{2} d x+\frac{1}{2} E I \int_{l_{\mathrm{b}}}^{l_{2}}\left(\frac{d^{2} w_{3}}{d x^{2}}\right)^{2} d x \text { for case } b
\end{array}\right.
$$

The energy loss $V_{2}$ due to lateral soil resistance is

$$
V_{2}=\left\{\begin{array}{l}
\int_{0}^{l_{\mathrm{b}}}\left|f_{\mathrm{b}} w_{1}\right| d x+\int_{l_{\mathrm{b}}}^{l_{1}}\left|f_{1} w_{2}\right| d x+\int_{l_{1}}^{l_{2}}\left|f_{1} w_{3}\right| d x \text { for case } a \\
\int_{0}^{l_{1}}\left|f_{\mathrm{b}} w_{1}\right| d x+\int_{l_{1}}^{l_{\mathrm{b}}}\left|f_{\mathrm{b}} w_{2}\right| d x+\int_{l_{\mathrm{b}}}^{l_{2}}\left|f_{1} w_{3}\right| d x \text { for case } b
\end{array}\right.
$$


The energy loss $V_{3}$ due to axial soil resistance is

$$
V_{3}=\int_{0}^{l_{\mathrm{b}}}\left|f_{A b} \bar{u}_{2}\right| d x+\int_{l_{\mathrm{b}}}^{l_{2}}\left|f_{A 1} \bar{u}_{3}\right| d x+\int_{l_{2}}^{l_{s}}\left|f_{A 1} \bar{u}_{1}\right| d x \quad \text { for both case } a \text { and case } b
$$

The axial compressive strain energy $V_{4}$ due to the axial compressive force is

$$
V_{4}=\frac{1}{2 E A} \int_{0}^{l_{s}} \bar{P}(x)^{2} d x \quad \text { for both case } a \text { and case } b
$$

while the total potential energy of the straight pipeline, namely before buckling, is given by

$$
V_{i}=\frac{1}{2 E A} \int_{0}^{l_{s}} P_{0}^{2} d x
$$

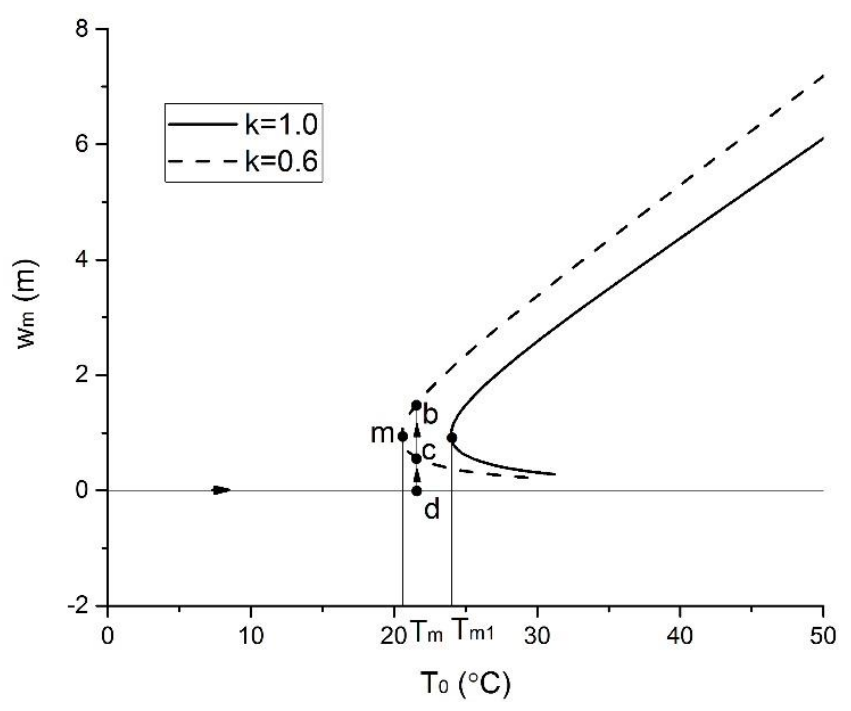

Fig. 3 Typical buckling path. $l_{b}=25 \mathrm{~m}$.

In Fig. 3, when $T_{0}$ is lower than $T_{m}$ only the trivial state $\left(w_{m}=0\right)$ exists and no lateral buckling occurs for $k=0.6$. However, when $T_{0}$ is larger than $T_{m}$, two lateral buckling states exist. Take $T_{0}=21.64{ }^{\circ} \mathrm{C}$, for example. When $T_{0}$ reaches $21.64{ }^{\circ} \mathrm{C}$, the pipeline will remain unbuckled in the absence of a disturbance or imperfection, corresponding to point $d$ in Fig. 3. However, the deformed states $b$ and $c$ are available as well and a sufficiently large disturbance may cause a jump from $d$ to one of these buckled states. Thus a jump occurs from $d$ to either $b$ or $c$ in Fig. 3. The total energy is calculated through Eq. (35) to determine the relative stability of the two branches.

The total energy of branches $m-b$ and $m-c$ for the lateral post-buckling state are denoted by $V_{b}$ and $V_{c}$, respectively. $V_{i b}$ and $V_{i c}$ are the total potential energies of the unbuckled pipeline with distributed buoyancy section of corresponding length $l_{s} . V_{b} / V_{i b}$ and $V_{c} / V_{i c}$ are illustrated in Fig. $4 . k=1$ in Fig. 4 represents the case of a pipeline without distributed buoyancy section.

All the values of $V_{b} / V_{i b}$ are less than those of $V_{c} / V_{i c}$, which means that the branch $m$ - $b$ is more stable than branch $m$ $c$. In addition, the value of $V_{c} / V_{i c}$ first increases slightly and then decreases with increasing temperature difference, while all the values of $V_{c} / V_{i c}$ are larger than 1, which means that branch $m$-c is less stable than the trivial solution. The value of $V_{b} / V_{i b}$ decreases with increasing temperature difference, which means that the branch $m-b$ becomes more stable with increasing temperature difference. $V_{b} / V_{i b}=1$ when the temperature difference reaches $T_{e}=21.50{ }^{\circ} \mathrm{C}$. For $T_{0}<T_{e}$, $V_{b} / V_{i b}$ is bigger than 1, which means that the trivial solution is more stable. For $T_{0}>T_{e}, V_{b} / V_{i b}$ is smaller than 1 , which means that the branch $m-b$ is more stable than the trivial state.

Fig. 3 also displays the buckling curve for the same pipeline without distributed buoyancy section. For $T_{0}>T_{m 1}$ two deformed states are available and an energy analysis shows that again the upper branch contains solutions with lower energy than the trivial state. A pipeline without distributed buoyancy section, therefore, would likely jump into a buckled state under a sufficiently large disturbance when $T_{0}$ becomes larger than $T_{m 1}$. This buckling would be sudden, without any warning signs, and could happen at any point of a long immobilised pipeline. This potentially dangerous scenario is avoided by using a distributed buoyancy section, which forces the pipeline into a deformed state at a specific point and at lower $T_{0}$ before the 
critical temperature $T_{m 1}$ is reached. In Fig. 3 the amplitude of lateral deflection will be larger in the case of a distributed buoyancy section (at the same temperature difference $T_{0}$ ). The effect of the distributed buoyancy section will be investigated in more detail in Section 3.2.

In Fig. 5 the composition of the total potential energy of the stable buckled pipeline is analysed, namely branch $m-b$. Both $V_{1} / V$ and $V_{2} / V$ first increase slightly and then reduce to less than $10 \%$ with increasing temperature difference. However, $V_{3} / V$ increases rapidly with temperature. The conclusion can be drawn that for large temperature difference the main energy loss is due to axial soil resistance. $V_{4} / V$ decreases rapidly to $50 \%$ at about $25^{\circ} \mathrm{C}$ first and then decreases slightly with increasing temperature difference. Thus, the total potential energy of the buckled pipeline at large $T_{0}$ consists mainly of $V_{3}$ and $V_{4}$.

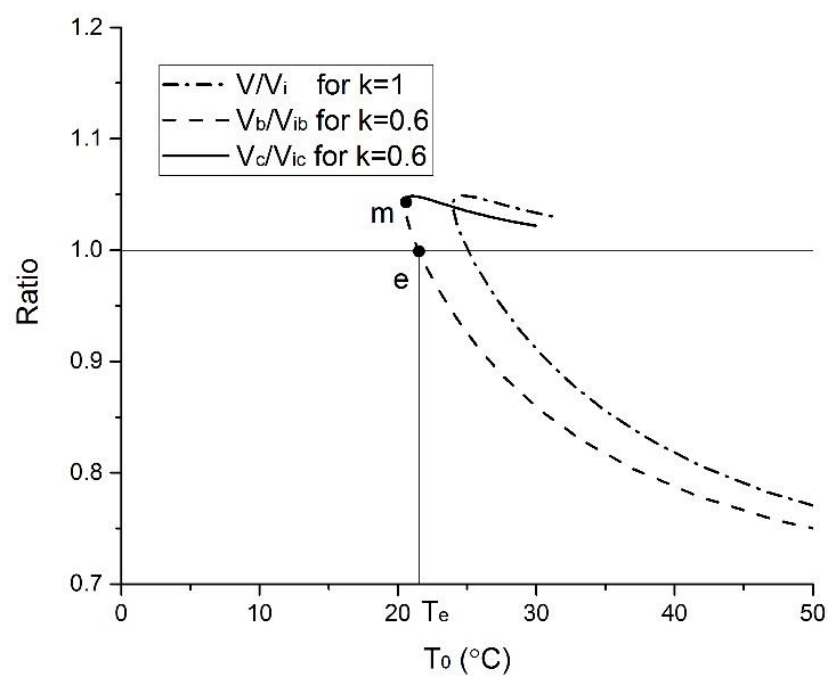

Fig. 4 Ratio of the energy between the buckled state and the pre-buckling state. $l_{b}=25 \mathrm{~m}$.

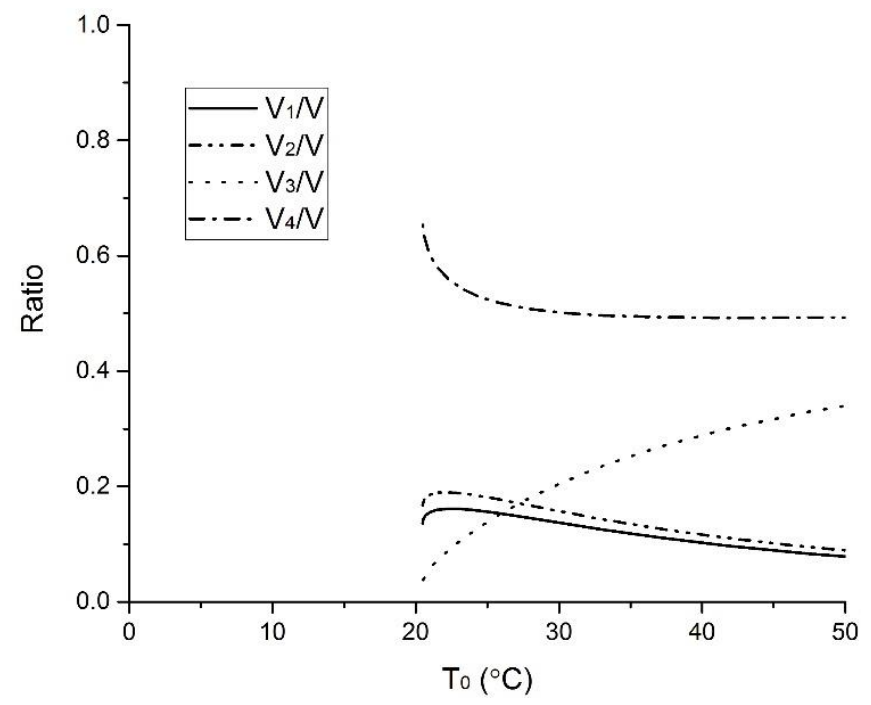

Fig. 5 Composition of the total potential energy of the buckled pipeline. $l_{b}=25 \mathrm{~m}$.

\subsection{Parameter study}

In this section, a typical pipeline with distributed buoyancy section resting on the seabed is analysed in the third lateral buckling mode. The deformed shapes and bending stresses along the pipeline with different $l_{b}$ and $k$ under the same temperature are analysed and discussed first. Then the properties of pipeline lateral buckling, such as the lateral buckling amplitude $w_{m}$, the axial compressive force $P$, the length of axial thermal expansion $u_{1}$ and the maximum axial compressive stress $\sigma_{m}$, are presented and analysed. Finally, the influence of $l_{b}$ and $k$ on the minimum critical temperature difference $T_{m}, w_{m}$ and $\sigma_{m}$ is discussed. The effect of the distributed buoyancy section is demonstrated by employing the analytical formulation developed in Section 2 taking the parameters in Table 1 as a realistic case study. In this section, all the analysis is based on branch $\mathrm{m}-\mathrm{b}$, namely the stable branch. The case $l_{b}=0 \mathrm{~m}$ and $k=1$ represents the situation of a pipeline 
without distributed buoyancy section, the results for which are similar to Hobbs's and Liu's results $[9,19]$.

Table 1. Design parameters.

\begin{tabular}{ccc}
\hline Parameter & Value & Unit \\
\hline External diameter $D$ & 323.9 & $\mathrm{~mm}$ \\
Wall thickness $t$ & 12.7 & $\mathrm{~mm}$ \\
Elastic modulus $E$ & 206 & $\mathrm{GPa}$ \\
Steel density $\rho$ & 7850 & $\mathrm{~kg} / \mathrm{m}^{3}$ \\
Coefficient of thermal expansion $\alpha$ & $1.1 \times 10^{-5}$ & $/{ }^{\circ} \mathrm{C}$ \\
Lateral friction coefficient $\mu_{L}$ & 0.5 & --- \\
Axial friction coefficient $\mu_{A}$ & 0.5 & --- \\
\hline
\end{tabular}

\subsubsection{Buckled configuration}

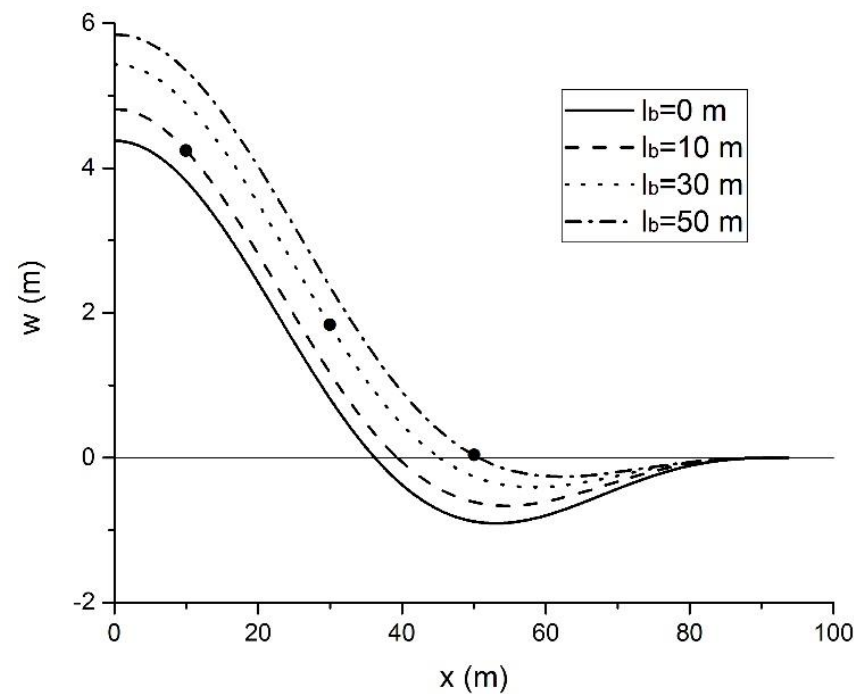

(a)

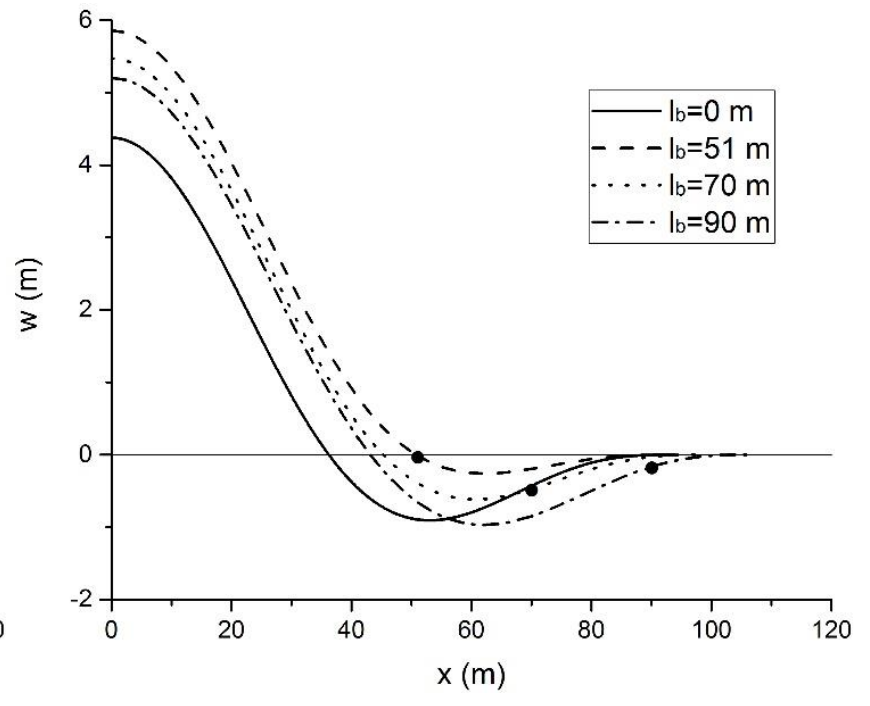

(b)

Fig. 6 Influence of $l_{b}$ on deformed shapes. (a) case $a$. (b) case $b . k=0.6 . T_{0}=40^{\circ} \mathrm{C}$. The dots indicate the boundary of the distributed buoyancy section.

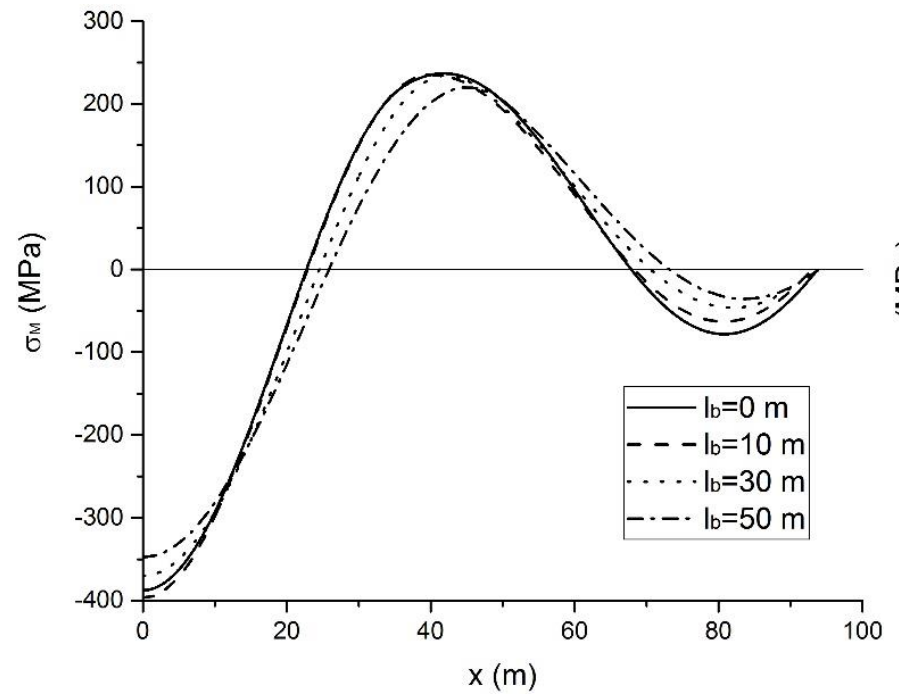

(a)

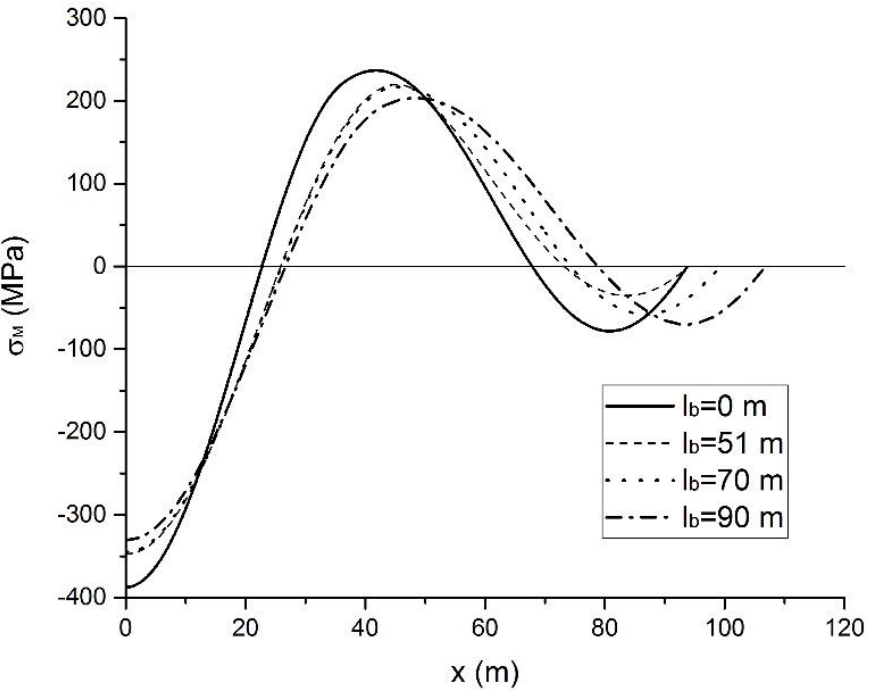

(b)

Fig. 7 Influence of $l_{b}$ on the bending stress. (a) case $a$. (b) case $b . k=0.6 . T_{0}=40{ }^{\circ} \mathrm{C}$.

The deformed shapes and corresponding bending stresses $\sigma_{M}$ along the buckled pipeline at different values of $l_{\mathrm{b}}$ under the same operating temperature difference $T_{0}$ are presented in Fig. 6 and Fig. 7, respectively. In Fig. 6, it is seen that a localised buckled shape is formed within a limited region in the middle of the pipeline due to the axial compressive force 
induced by the temperature difference. This shape consists of half a primary lobe in the positive direction $(w>0)$ and a secondary lobe in the negative direction $(w<0)$ for half a buckled pipeline. In Fig. 6 (a), it is seen that the three cases with $k=0.6$ have $l_{\mathrm{b}}<l_{1}$ and therefore belong to case $a$. By contrast, the cases in Fig. 6 (b) have $l_{1}<l_{\mathrm{b}}<l_{2}$ and therefore belong to case $b$. The lateral displacement amplitude is attained at $x=0 \mathrm{~m}$ for each case. It is clear that $l_{\mathrm{b}}$ has great influence on the deformed shapes of lateral buckling. The primary lobe for the case of $l_{\mathrm{b}}=0 \mathrm{~m}$ is smaller than that for all the cases of $l_{\mathrm{b}}>0 \mathrm{~m}$, for both case $a$ and case $b$. For case $a$, the primary lobe increases with increasing $l_{\mathrm{b}}$, while the secondary lobe decreases with increasing $l_{\mathrm{b}}$. However, for case $b$, the primary lobe decreases with increasing $l_{\mathrm{b}}$, while the secondary lobe increases with increasing $l_{\mathrm{b}}$.

In Fig. 7 it is seen that there are three maxima of bending stress in either the positive or negative direction. The maximum bending stress is attained at $x=0 \mathrm{~m}$, so the integrity of the pipeline will be lost first at the location $x=0 \mathrm{~m}$. The amplitude of bending stress at $x=0 \mathrm{~m}$ for the case $l_{\mathrm{b}}=10 \mathrm{~m}$ is larger than that for the case $l_{\mathrm{b}}=0 \mathrm{~m}$, which will decrease with further increase of $l_{\mathrm{b}}$ for case $a$, as shown in Fig. 7 (a). For the cases $l_{\mathrm{b}}=51 \mathrm{~m}$ and $l_{\mathrm{b}}=70 \mathrm{~m}$, the maximum bending stress at $x=0 \mathrm{~m}$ stays almost the same, which is smaller than that for $l_{\mathrm{b}}=0 \mathrm{~m}$ and bigger than that for $l_{\mathrm{b}}=90 \mathrm{~m}$. This variation of the maximum bending stress at $x=0 \mathrm{~m}$ is complicated. Since it is important in practice, its variation with $l_{\mathrm{b}}$ is analysed further in Section 3.2.2 below.

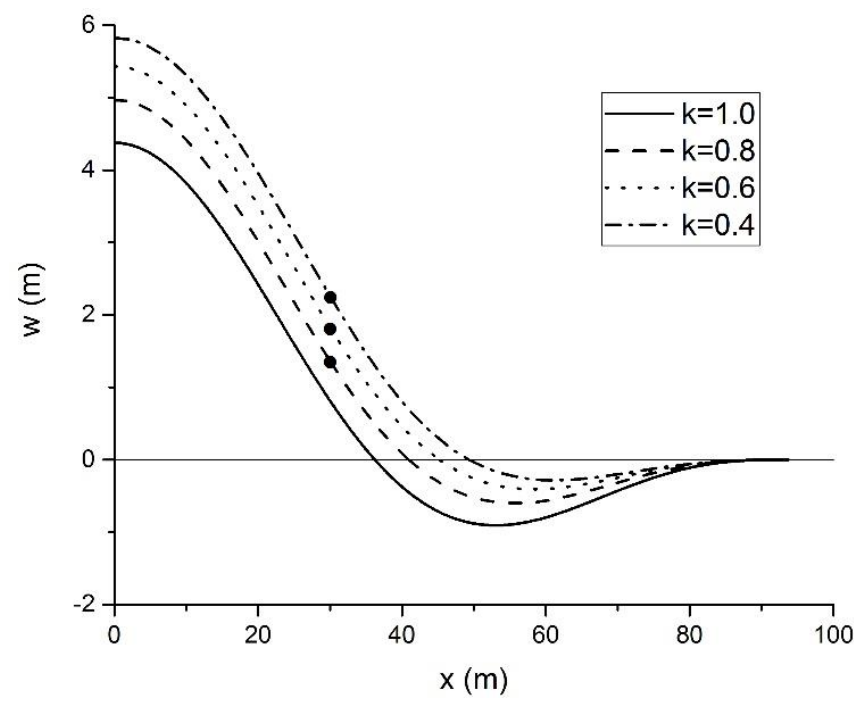

(a)

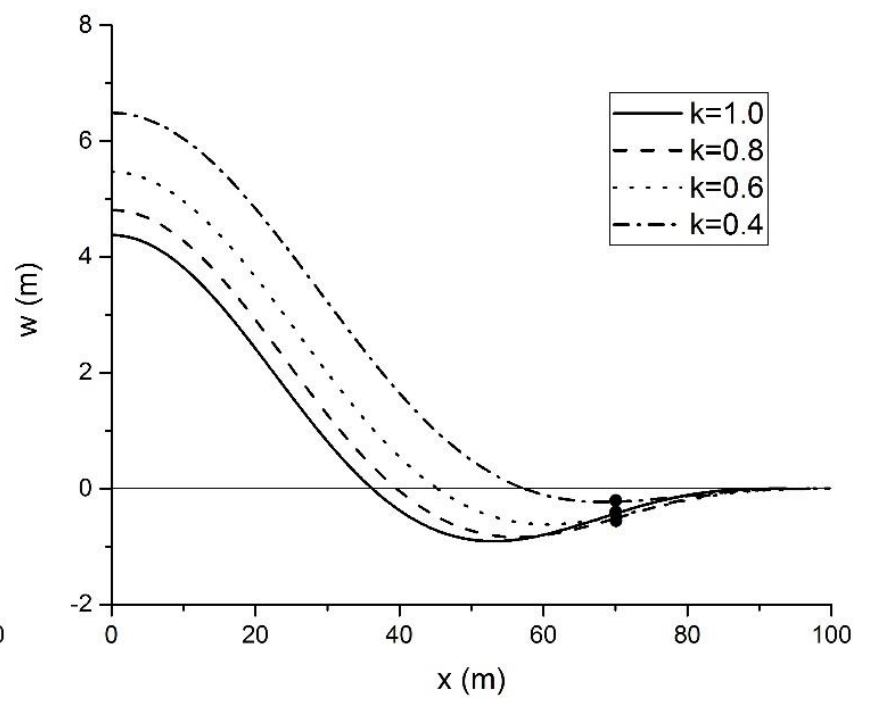

(b)

Fig. 8 Influence of $k$ on deformed shapes. (a) case $a . l_{b}=30 \mathrm{~m}$. (b) case $b . l_{b}=70 \mathrm{~m} . T_{0}=40{ }^{\circ} \mathrm{C}$. The dots indicate the boundary of the distributed buoyancy section.

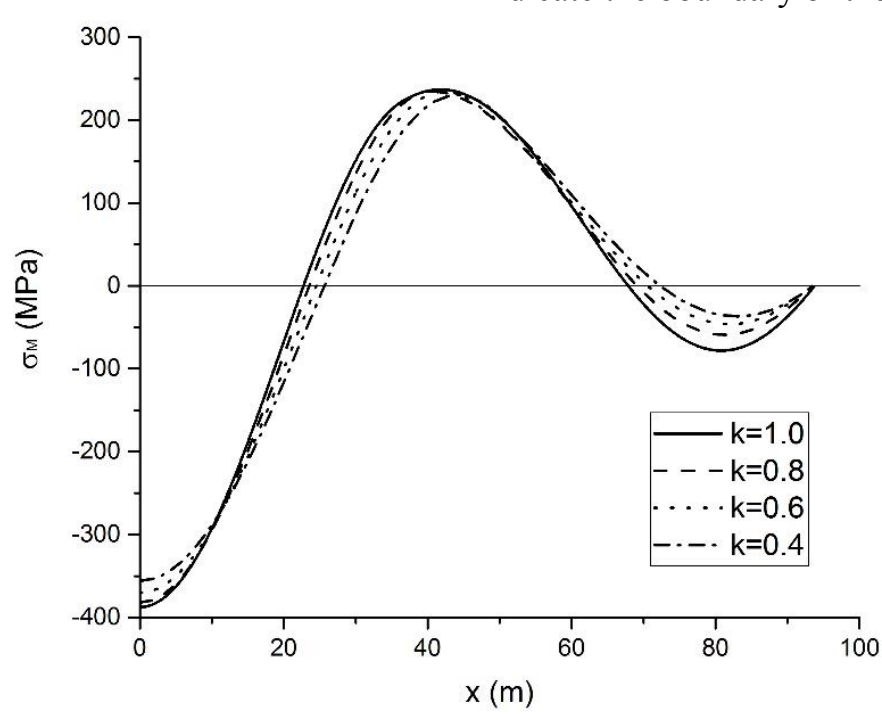

(a)

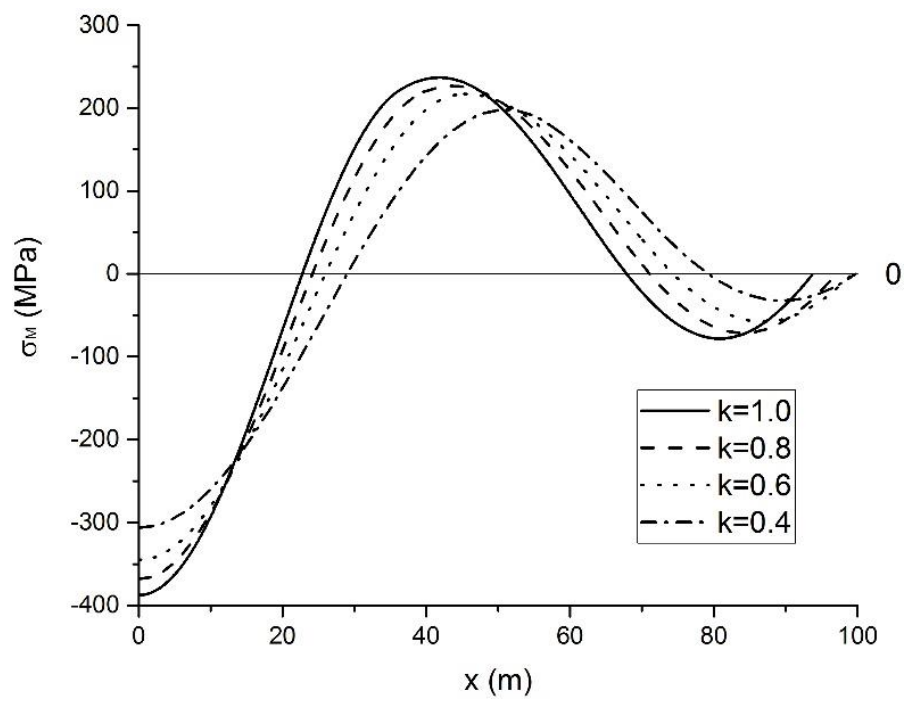

(b)

Fig. 9 Influence of $k$ on the bending stress. (a) case $a . l_{b}=30 \mathrm{~m}$. (b) case $b . l_{b}=70 \mathrm{~m} . T_{0}=40^{\circ} \mathrm{C}$. 
The deformed shapes and the corresponding bending stresses $\sigma_{M}$ along the buckled pipeline at different values of $k$ under the same operating temperature difference are presented in Fig. 8 and Fig. 9, respectively. It is clear that $k$ has great influence on the deformed shapes of lateral buckling. The maximum displacement is attained at $x=0 \mathrm{~m}$ for each value of $k$. In Fig. 8 , the primary lobe for the case of $k=1$ is smaller than that for all the cases of $k<1$, for both case $a$ and case $b$, while the secondary lobe is larger. Under decreasing $k$, both the buckled region and the lateral deflection of the primary lobe increase. However, the secondary lobe shrinks with decreasing $k$, as shown in Fig. 8. In Fig. 9 it is seen that the maximum bending stress is attained at $x=0 \mathrm{~m}$ for each value of $k$, so the integrity of the pipeline will be lost first at the location $x=$ $0 \mathrm{~m}$. For case $a$, the value of $k$ does not have much influence on the distribution of the bending stress, as shown in Fig. 9 (a). However, for case $b$, in Fig. 9 (b), the location of maximum bending stress moves away from the centre of the buckled pipeline with decreasing $k$. All local maxima of bending stress decrease with decreasing $k$ for both case $a$ and case $b$, but the amount of decrease for case $a$ is smaller than that for case $b$.

\subsubsection{Typical buckling behaviour}

In this section, typical buckling behaviour for pipelines with distributed buoyancy section is analysed. All the analysis is based on the stable branch $m-b$.

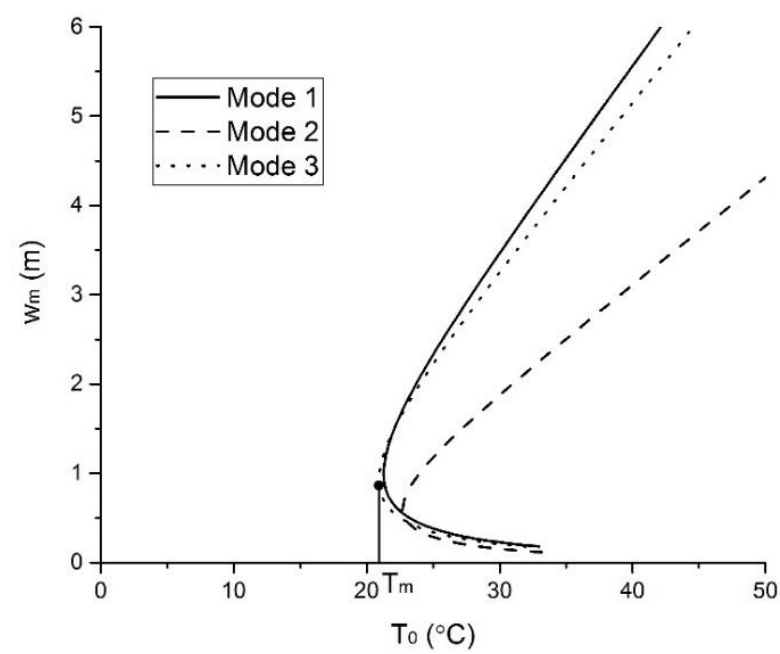

Fig. 10 Comparison between different lateral buckling modes.

A comparison between different lateral buckling modes is shown in Fig. 10. It is observed that the minimum critical temperature difference $T_{\mathrm{m}}$ for mode 3 is the smallest. This is the reason why the third mode is selected as the basis to derive the analytical solution in this paper. The minimum critical temperature difference for mode 1 is slightly larger than that for mode 3, while the minimum critical temperature difference for mode 2 is the largest. However, the lateral displacement amplitude in the post-buckling stage for mode 2 is the smallest under the same temperature difference.

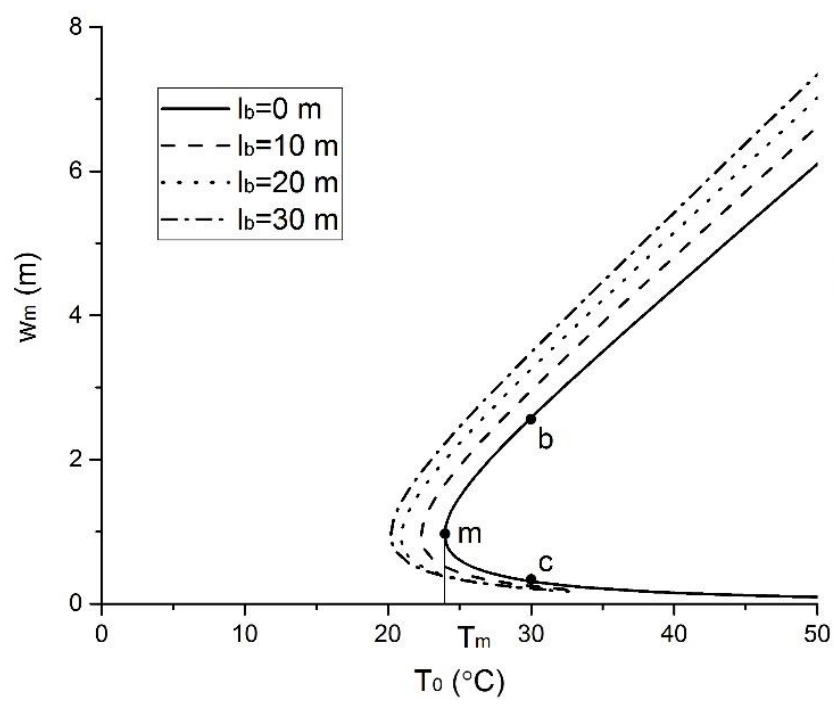

(a)

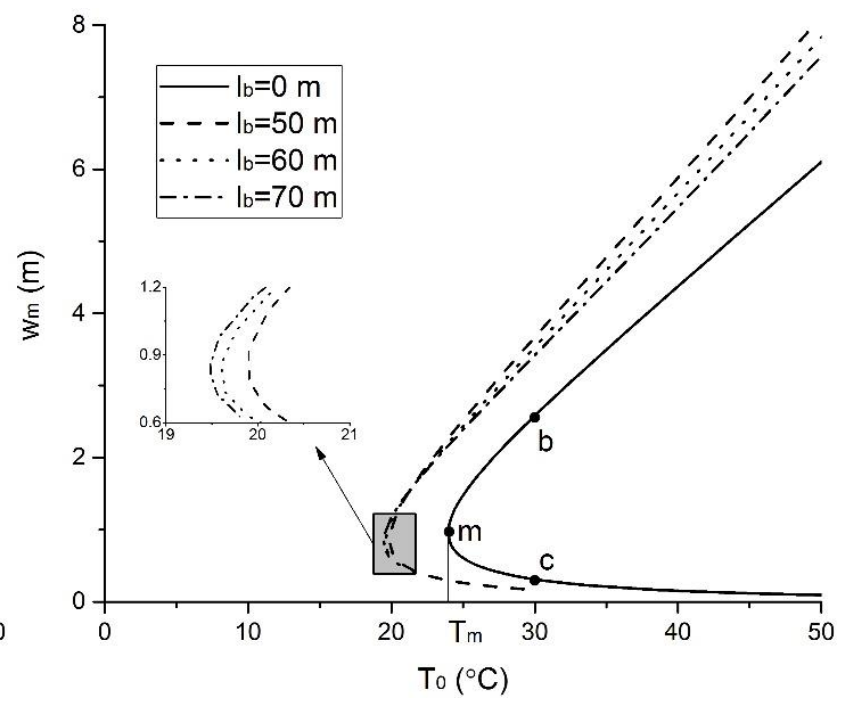

(b) 
Fig. 11 Lateral displacement amplitude $w_{m}$. (a) case $a$. (b) case $b . k=0.6$.

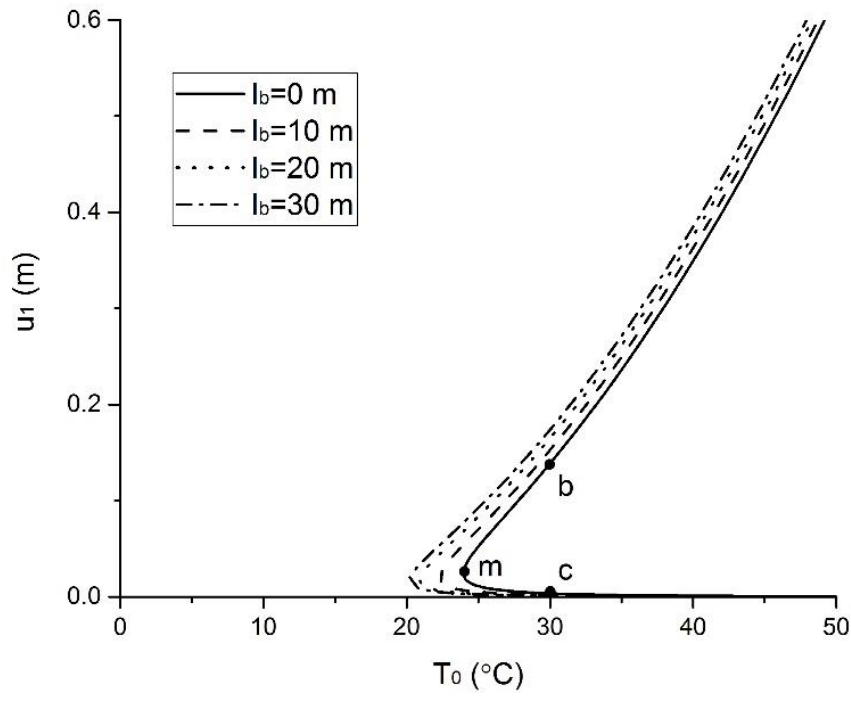

(a)

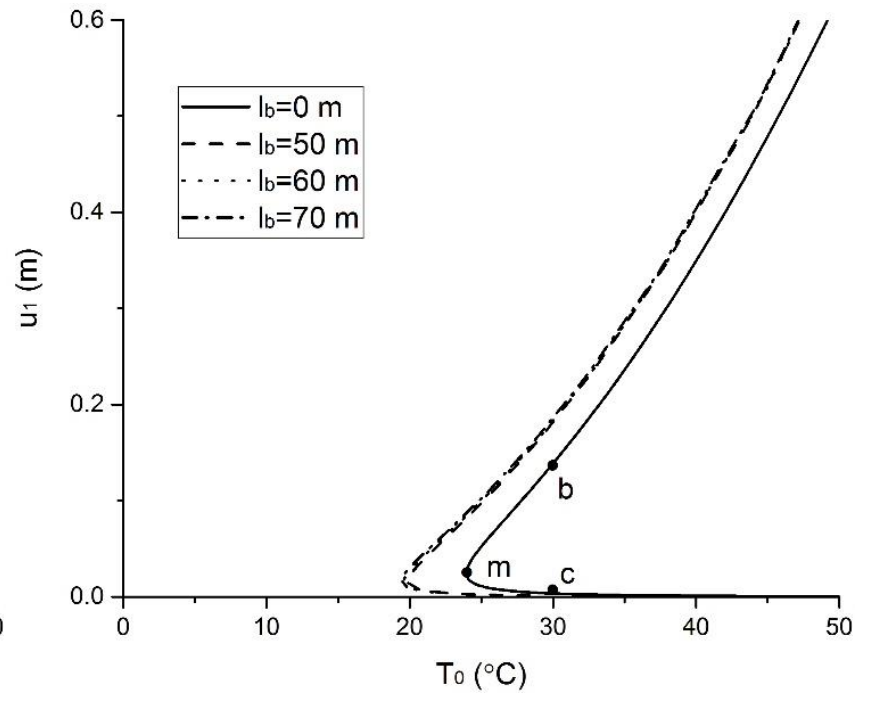

(b)

Fig. 12 Length of the axial thermal expansion $u_{1}$. (a) case $a$. (b) case $b . k=0.6$.

The relationships between lateral buckling amplitude $w_{m}$ and total temperature difference $T_{0}$ at different values of $l_{b}$ are shown in Fig. 11, while the relationships between the length of axial thermal expansion $u_{1}$ and total temperature difference $T_{0}$ at different $l_{b}$ are shown in Fig. 12. In Fig. 11 the minimum critical temperature difference $T_{m}$ decreases with increasing $l_{b}$ for both case $a$ and case $b$, which means that it will be easier for pipelines to have lateral buckling with larger $l_{b}$. But the decreased amount of $T_{m}$ for case $b$ is smaller than that for case $a$ with the same increase in $l_{b}$, which will be analysed in more detail in Section 3.2.3 below. After lateral buckling happens, namely for branch m-b, the lateral buckling amplitude $w_{m}$ increases under increasing total temperature difference at a specific $l_{b}$ for both case $a$ and case $b$, as shown in Fig. 11. This is because the length of the axial thermal expansion $u_{1}$ increases with increasing total temperature difference (see Fig. 12), which means that more $u_{1}$ will feed into the buckled region, leading to a more significant lateral buckle. The rate of increase of $w_{m}$ for case $a$ stays almost the same, as shown in Fig. 12 (a). However, for case $b$, in Fig. 12 (b), the rate of increase of $w_{m}$ decreases with increasing $l_{b}$. Also, $w_{m}$ increases with increasing $l_{b}$ for case $a$ with the same total temperature difference. The reason is that the primary lobe grows and the secondary lobe shrinks under increasing $l_{\mathrm{b}}$ (see Fig. 6 (a)) and $u_{1}$ increases with increasing $l_{b}$ (see Fig. 12 (a)) at the same total temperature difference for case $a$. By contrast, $w_{m}$ decreases with increasing $l_{b}$ for case $b$ at the same total temperature difference: according to Fig. 6 (b), the primary lobe shrinks and the secondary lobe grows under increasing $l_{\mathrm{b}}$ for case $b$, so with almost the same axial thermal expansion (see Fig. 12 (b)), the rate of increase of $w_{m}$ decreases with increasing $l_{b}$ for case $b$.

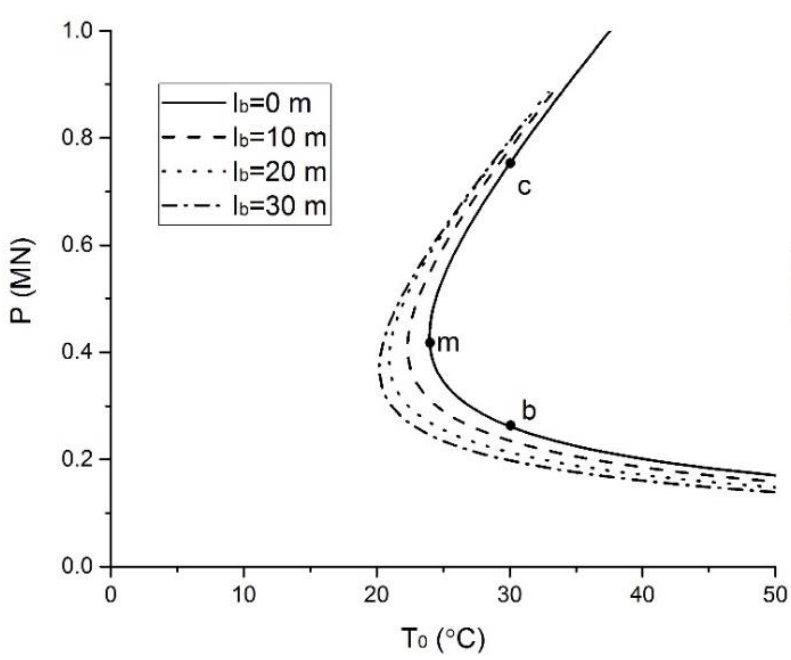

(a)

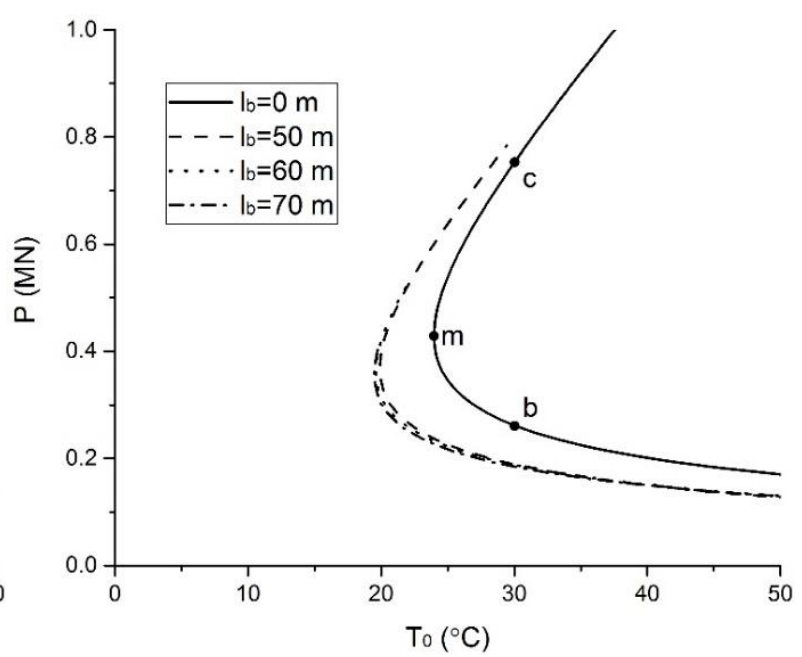

(b) 
Fig. 13 Axial compressive force $P$. (a) case $a$. (b) case $b . k=0.6$.

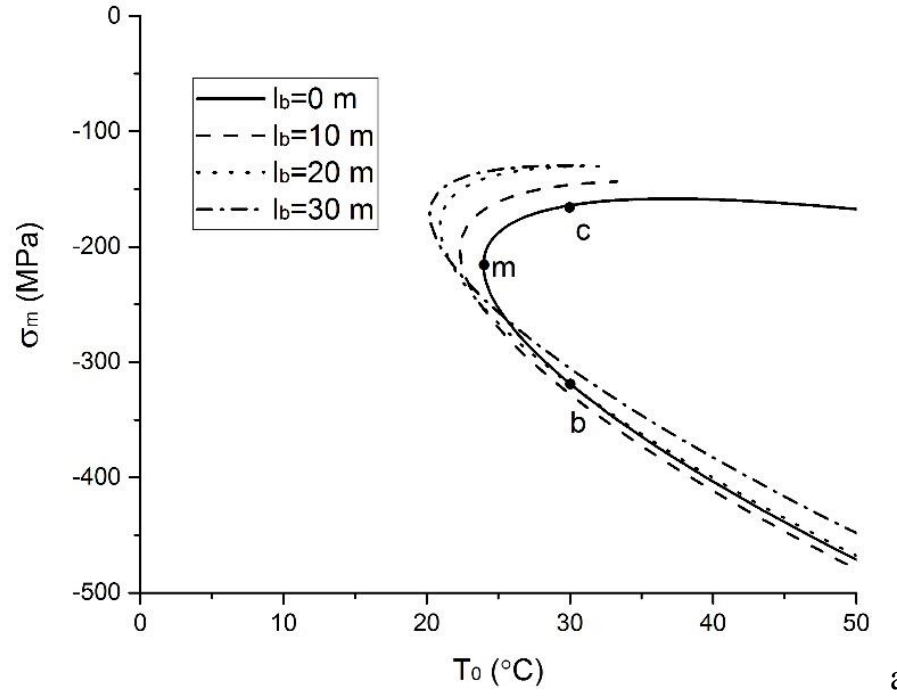

(a)

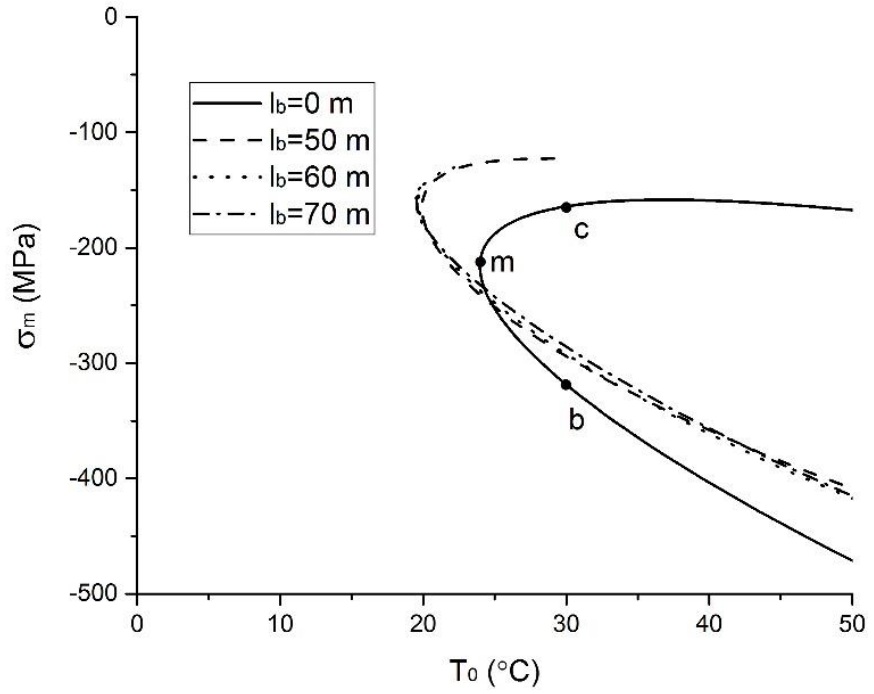

(b)

Fig. 14 Maximum axial compressive stress $\sigma_{m}$. (a) case $a$. (b) case $b . k=0.6$.

The relationships between axial compressive force $P$ and total temperature difference $T_{0}$ at different values of $l_{b}$ are shown in Fig. 13. It is seen in Fig. 13 that the axial compressive force $P$ decreases with increasing total temperature difference $T_{0}$ for each specific $l_{b}$. This shows that the process of lateral buckling results in an axial compressive force reduction under increasing $T_{0}$. The axial compressive force $P$ decreases with increasing $l_{b}$ for both case $a$ and case $b$ under the same total temperature difference. But the influence of $l_{b}$ on $P$ is very small for case $b$, as shown in Fig. 13 (b). Thus, the pipeline will be more stable, because of smaller axial compressive force $P$ in the post-buckling stage, if $l_{b}$ is larger.

The relationships between the maximum axial compressive stress $\sigma_{m}$ and the total temperature difference $T_{0}$ at different values of $l_{b}$ are shown in Fig. 14. It is seen that the maximum axial compressive stress $\sigma_{m}$ increases (in absolute value) with increasing total temperature difference $T_{0}$ for given $l_{b}$. The rate of increase decreases with increasing $l_{b}$ for case $a$, while the rate of increase stays almost the same under varying $l_{b}$ for case $b$. Taking $T_{0}=40{ }^{\circ} \mathrm{C}$ for example, the maximum axial compressive stress $\sigma_{m}$ first increases for $l_{b}=10 \mathrm{~m}$, then decreases with increasing $l_{b}$ for case $a$ under the same total temperature difference. Meanwhile, the influence of $l_{b}$ for case $b$ is smaller than that for case $a$ for the same increase of $l_{b}$. So the selection of the length $l_{b}$ is very important. The influence of $l_{b}$ on $\sigma_{m}$ is complicated and will be discussed in Section 3.2.4 below.

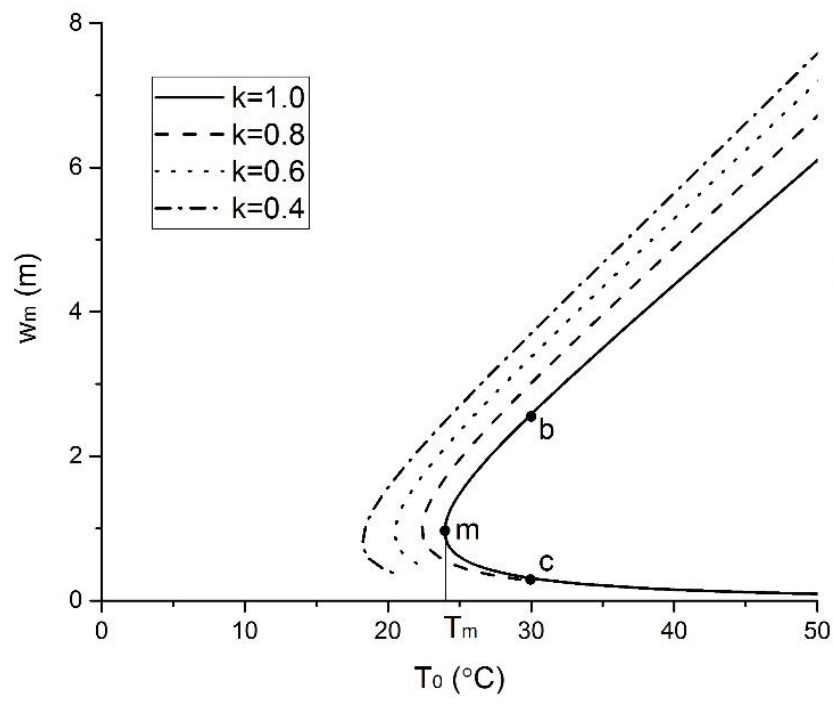

(a)

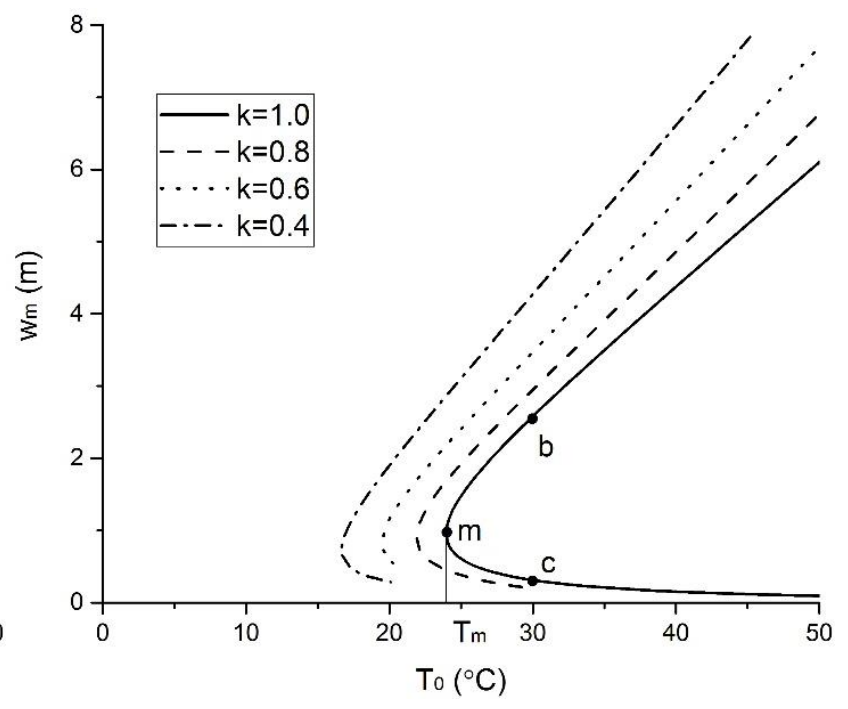

(b)

Fig. 15 Lateral displacement amplitude $w_{m}$. (a) case $a . l_{b}=25 \mathrm{~m}$. (b) case $b . l_{b}=65 \mathrm{~m}$. 


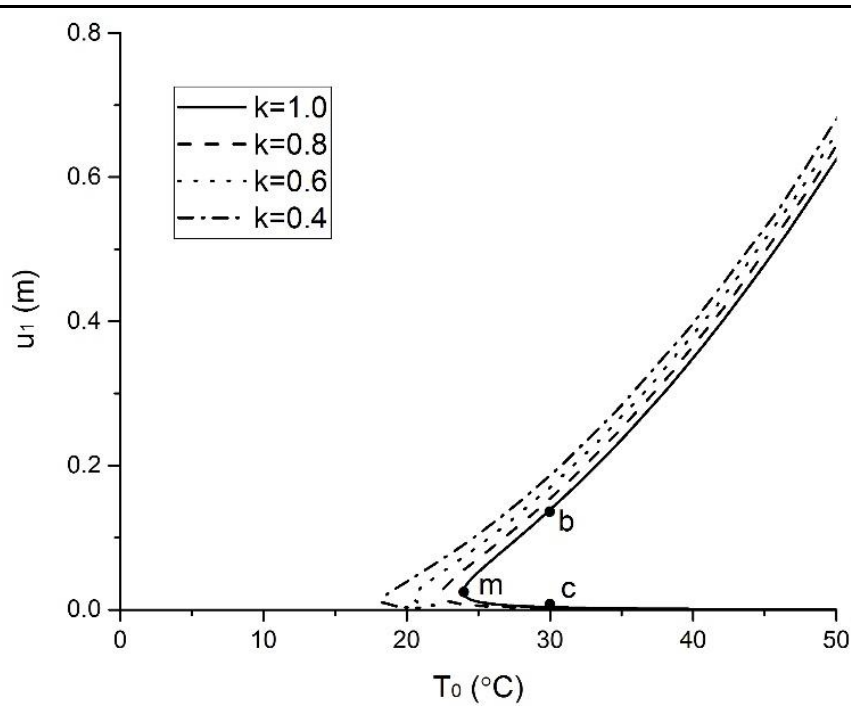

(a)

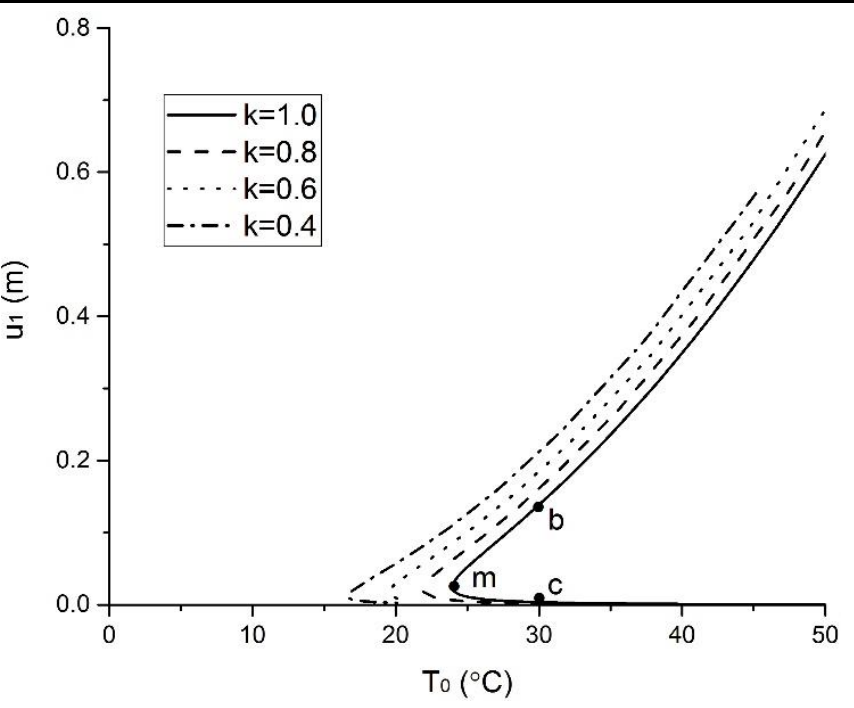

(b)

Fig. 16 Length of axial thermal expansion $u_{1}$. (a) case $a . l_{b}=25 \mathrm{~m}$. (b) case $b . l_{b}=65 \mathrm{~m}$.

The relationships between the lateral buckling amplitude $w_{m}$ and the total temperature difference $T_{0}$ at different values of $k$ are shown in Fig. 15, while the relationships between the length of the axial thermal expansion $u_{1}$ and the total temperature difference $T_{0}$ at different values of $k$ are shown in Fig. 16. In Fig. 15 the minimum critical temperature difference $T_{m}$ decreases with decreasing $k$ for both case $a$ and case $b$, which means that it will be easier for pipelines to have lateral buckling if $k$ is smaller. After lateral buckling happens, namely for branch $\mathrm{m}-\mathrm{b}$, the lateral buckling amplitude $w_{m}$ increases under increasing total temperature difference at given $k$ for both case $a$ and case $b$, as shown in Fig. 15. This is because the length of axial thermal expansion $u_{1}$ increases with increasing total temperature difference (see Fig. 16), which means that more $u_{1}$ will feed into the buckled region. The rate of increase of $w_{m}$ for case $a$ stays almost the same, while for case $b$ it increases with decreasing $k$. Meanwhile, $w_{m}$ increases with decreasing $k$ at the same total temperature difference for both case $a$ and case $b$. The reason is that the primary lobe grows and the secondary lobe shrinks under decreasing $k$ (see Fig. 8) and $u_{1}$ increases with decreasing $k$ (see Fig. 16) at the same total temperature difference for both case $a$ and case $b$.

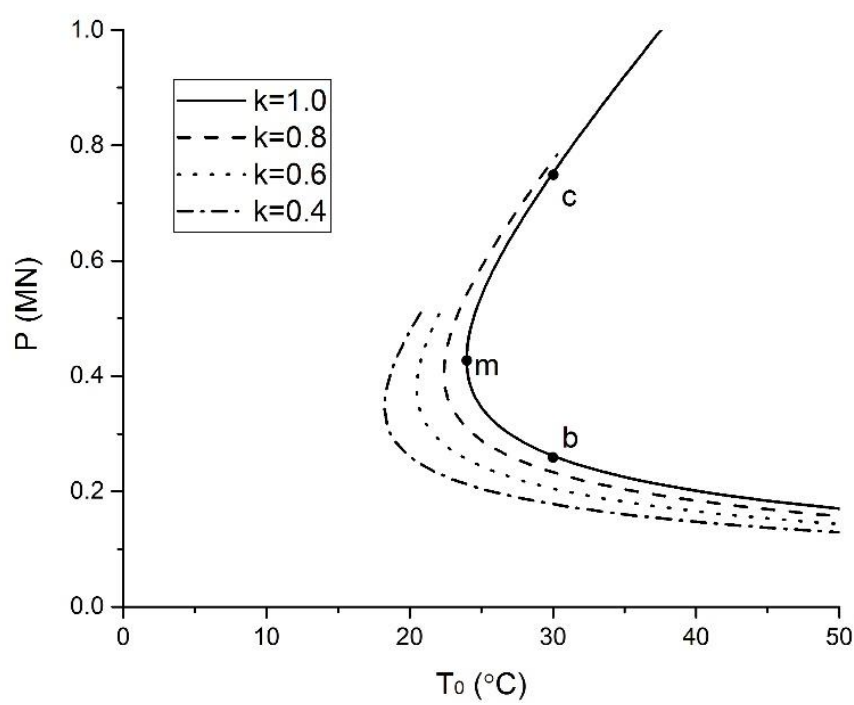

(a)

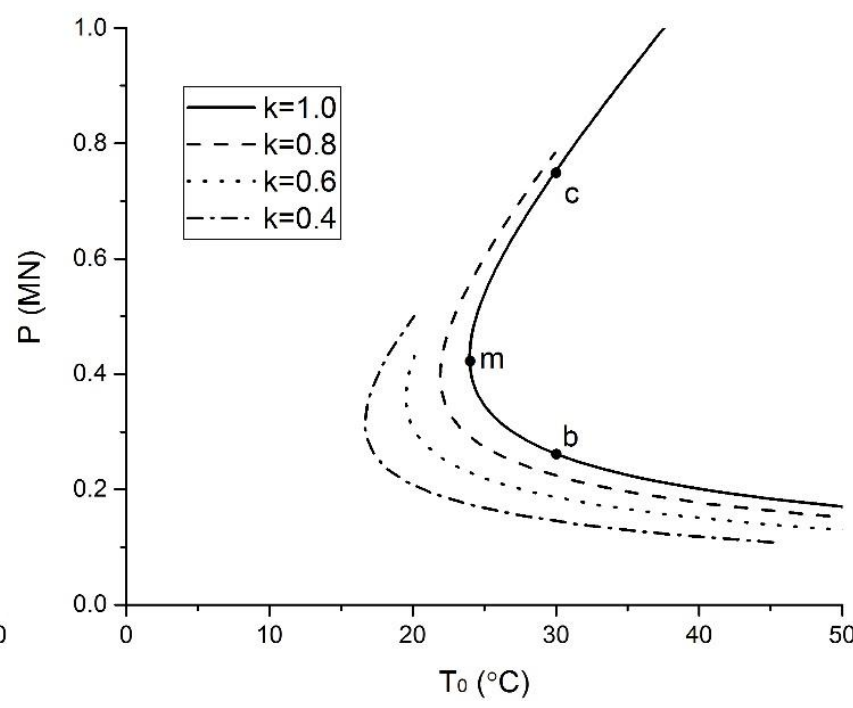

(b)

Fig. 17 Axial compressive force $P$. (a) case $a . l_{b}=25 \mathrm{~m}$. (b) case $b . l_{b}=65 \mathrm{~m}$. 


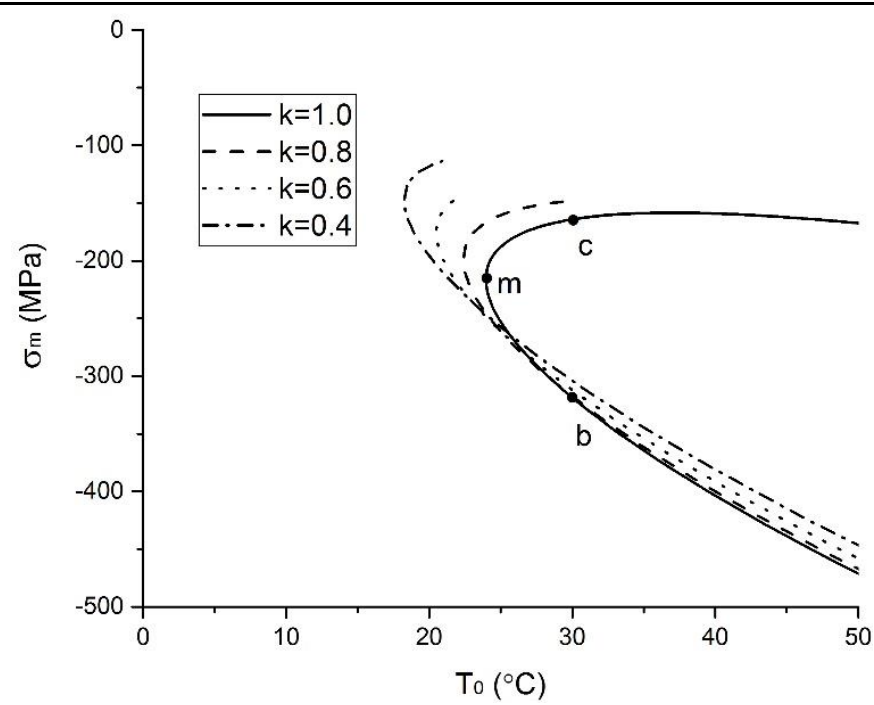

(a)

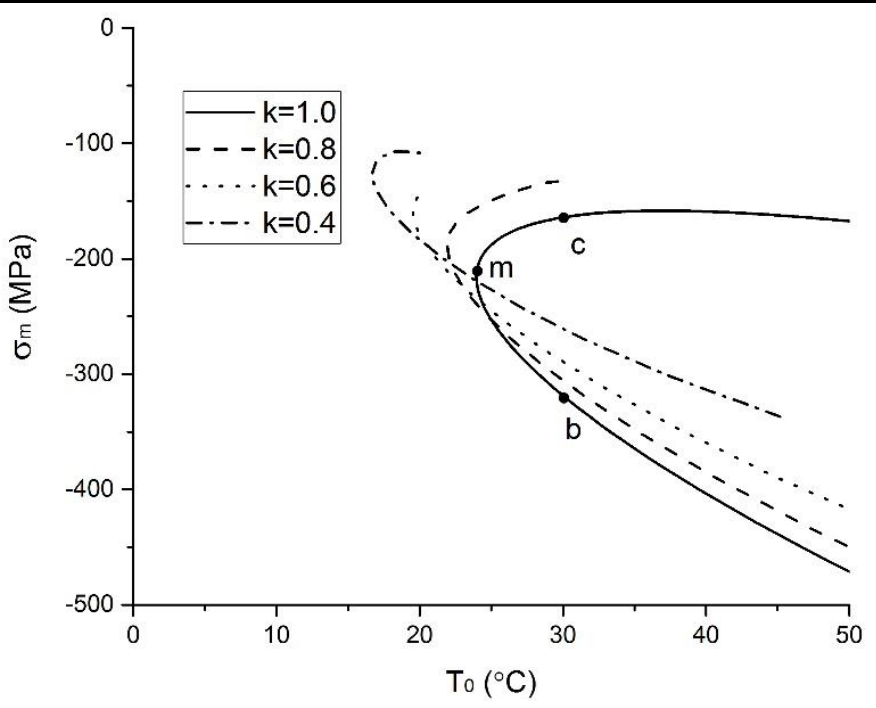

(b)

Fig. 18 Maximum axial compressive stress $\sigma_{m}$. (a) case $a . l_{b}=25 \mathrm{~m}$. (b) case $b . l_{b}=65 \mathrm{~m}$.

The relationships between axial compressive force $P$ and total temperature difference $T_{0}$ at different values of $k$ are shown in Fig. 17. It is seen that the axial compressive force $P$ decreases with increasing total temperature difference $T_{0}$ for each specific $k$. This shows that the process of lateral buckling results in an axial compressive force reduction under increasing $T_{0}$. The axial compressive force $P$ decreases with decreasing $k$ for both case $a$ and case $b$ at the same total temperature difference. Thus, the pipeline will be more stable, because of smaller axial compressive force $P$ in the postbuckling stage, if $k$ is smaller.

The relationships between the maximum axial compressive stress $\sigma_{m}$ and the total temperature difference $T_{0}$ at different values of $k$ are shown in Fig. 18. It is seen that the maximum axial compressive stress $\sigma_{m}$ increases (in absolute value) with increasing total temperature difference $T_{0}$ for given $k$. The rate of increase of $\sigma_{m}$ stays almost the same for different values of $k$ for case $a$, as shown in Fig. 18 (a). However, the rate of increase of $\sigma_{m}$ decreases with decreasing $k$ for case $b$ (see Fig. 18 (b)). For both case $a$ and case $b, \sigma_{m}$ decreases with decreasing $k$ at the same total temperature difference, but the influence of $k$ for case $b$ is larger than that for case $a$ for the same decrease of $k$.

\subsubsection{Minimum critical temperature difference}

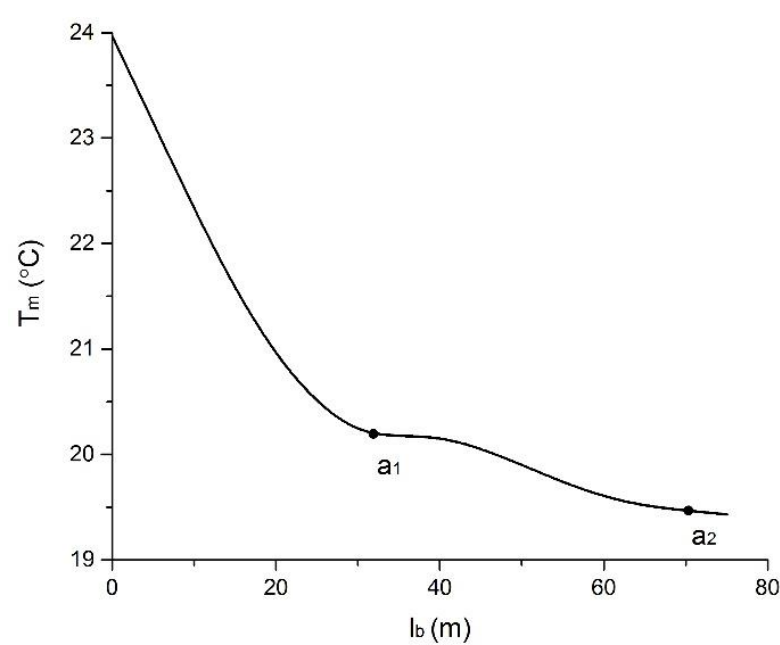

(a)

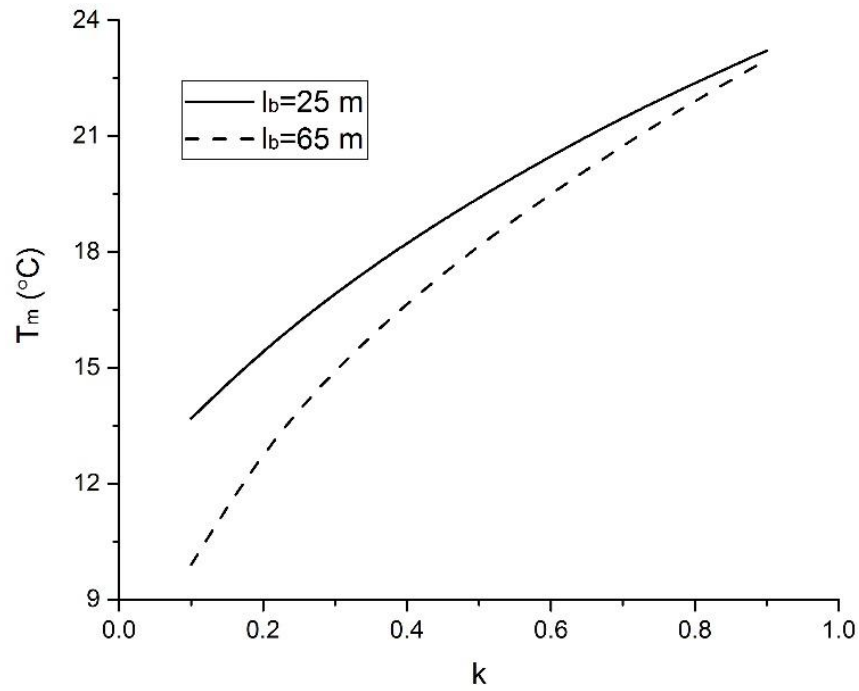

(b)

Fig. 19 Minimum critical temperature difference $T_{\mathrm{m}}$. (a) Influence of $l_{b}$ at $k=0.6$. (b) Influence of $k$ at $l_{b}=25 \mathrm{~m}$ (case $a$ ) and $l_{b}=65 \mathrm{~m}$ (case $b$ ).

The influence of the distributed buoyancy section on the minimum critical temperature difference $T_{\mathrm{m}}$ is illustrated in Fig. 19 , while the influence of the distributed buoyancy section on the lengths $l_{1}$ and $l_{2}$ corresponding to $T_{\mathrm{m}}$ is presented in 
Fig. 20. The result in Fig. 20 is used to judge whether the solution belongs to case $a$ or case $b$. In Fig. 19 (a) and Fig. 20 (a) two important points, namely $a_{1}$ and $a_{2}$, represent the boundary of case $a$ and case $b$. In Fig. 19 (a), for $l_{b}<l_{b}\left(a_{1}\right)$ we have $l_{b}<l_{1}$, so the solution belongs to case $a$, while for $l_{b}\left(a_{1}\right)<l_{b}<l_{b}\left(a_{2}\right)$, we have $l_{1}<l_{b}<l_{2}$, so the solution belongs to case $b$. When $l_{b}$ increases from $0 \mathrm{~m}$ to $30 \mathrm{~m}, T_{\mathrm{m}}$ decreases from $23.97{ }^{\circ} \mathrm{C}$ to $20.21{ }^{\circ} \mathrm{C}$ (a rate of decrease of $0.1253{ }^{\circ} \mathrm{C} / \mathrm{m}$ ). When $l_{b}$ increases from $30 \mathrm{~m}$ to $40 \mathrm{~m}, T_{\mathrm{m}}$ decreases from $20.21{ }^{\circ} \mathrm{C}$ to $20.17{ }^{\circ} \mathrm{C}$ (a rate of decrease of $0.0040{ }^{\circ} \mathrm{C} / \mathrm{m}$ ). When $l_{b}$ increases from $40 \mathrm{~m}$ to $70 \mathrm{~m}, T_{\mathrm{m}}$ decreases from $20.17{ }^{\circ} \mathrm{C}$ to $19.47{ }^{\circ} \mathrm{C}$ (a rate of decrease of $0.0233{ }^{\circ} \mathrm{C} / \mathrm{m}$ ). So it can be concluded that increasing $l_{b}$ has a more favourable effect on the decrease of $T_{\mathrm{m}}$ for case $a$ than for case $b$. From Fig. 20 (b) it can be deduced that for $l_{b}=25 \mathrm{~m}$ we have $l_{b}<l_{1}$ for all $k$ such that $0.1<k<0$.9. So the case $l_{b}=25 \mathrm{~m}$ in Fig. 19 (b) belongs to case $a$. On the other hand, for $l_{b}=65 \mathrm{~m}$ we have $l_{1}<l_{b}<l_{2}$ for all $k$ such that $0.1<k<0.9$, so the case $l_{b}=65 \mathrm{~m}$ in Fig. 19 (b) belongs to case $b$. Fig. 19 (b) shows that $T_{\mathrm{m}}$ decreases with decreasing $k$ for both $l_{b}=25 \mathrm{~m}$ and $l_{b}=65 \mathrm{~m}$, with the rate of decrease larger for $l_{b}=65 \mathrm{~m}$ than for $l_{b}=25 \mathrm{~m}$.

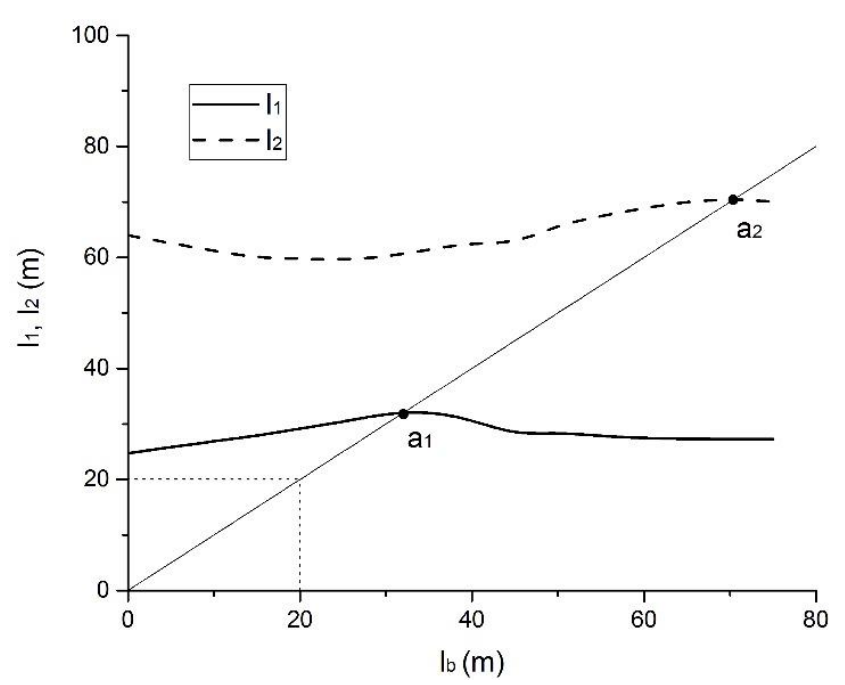

(a)

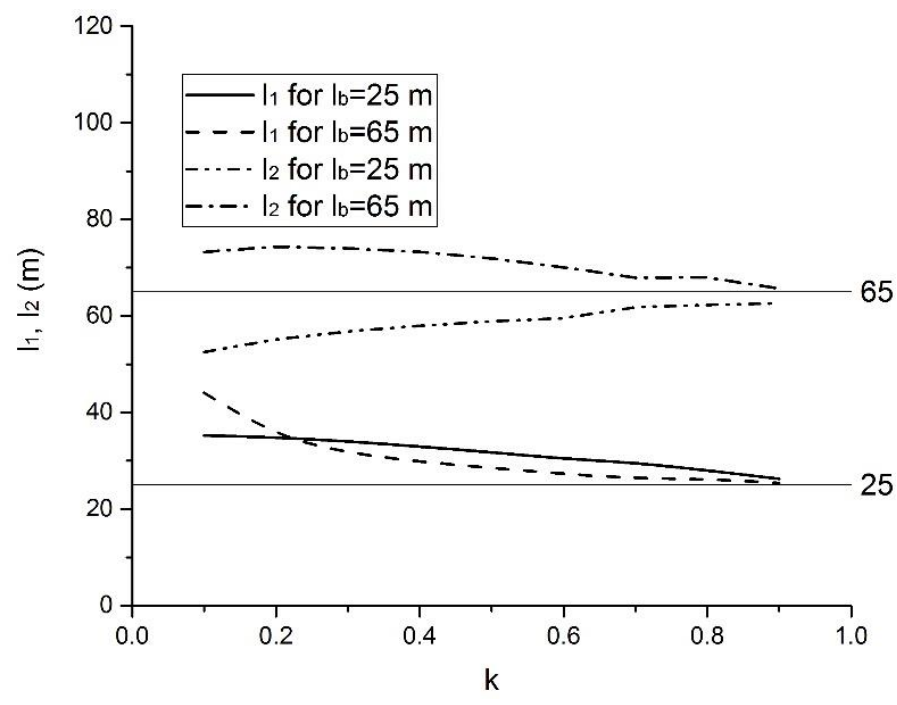

(b)

Fig. 20 Lengths $l_{1}$ and $l_{2}$ corresponding to $T_{\mathrm{m}}$. (a) $k=0.6$. (b) $l_{b}=25 \mathrm{~m}$ (case $a$ ) and $l_{b}=65 \mathrm{~m}$ (case $b$ ).

\subsubsection{Influence of distributed buoyancy section on $w_{m}$ and $\sigma_{m}$}

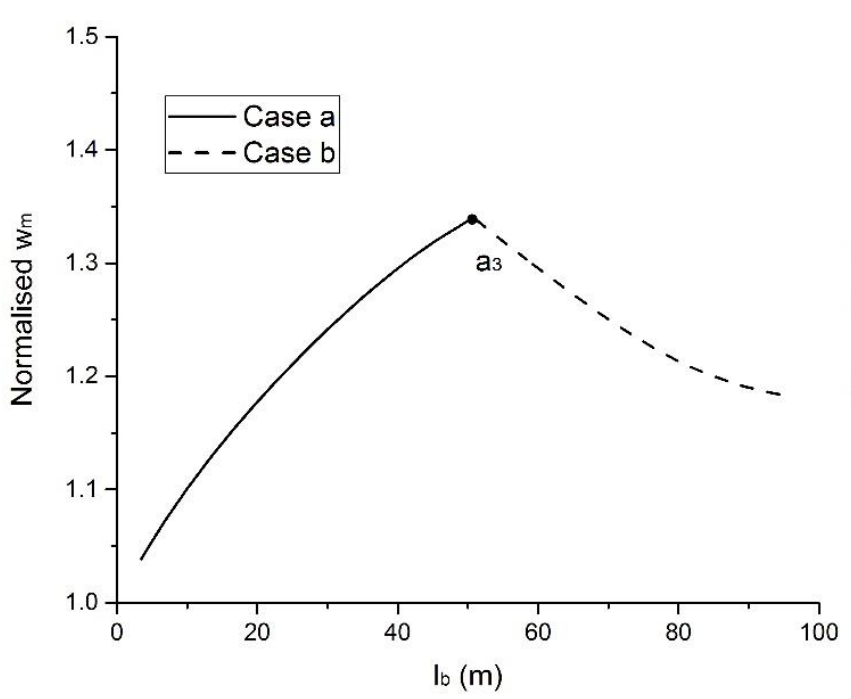

(a)

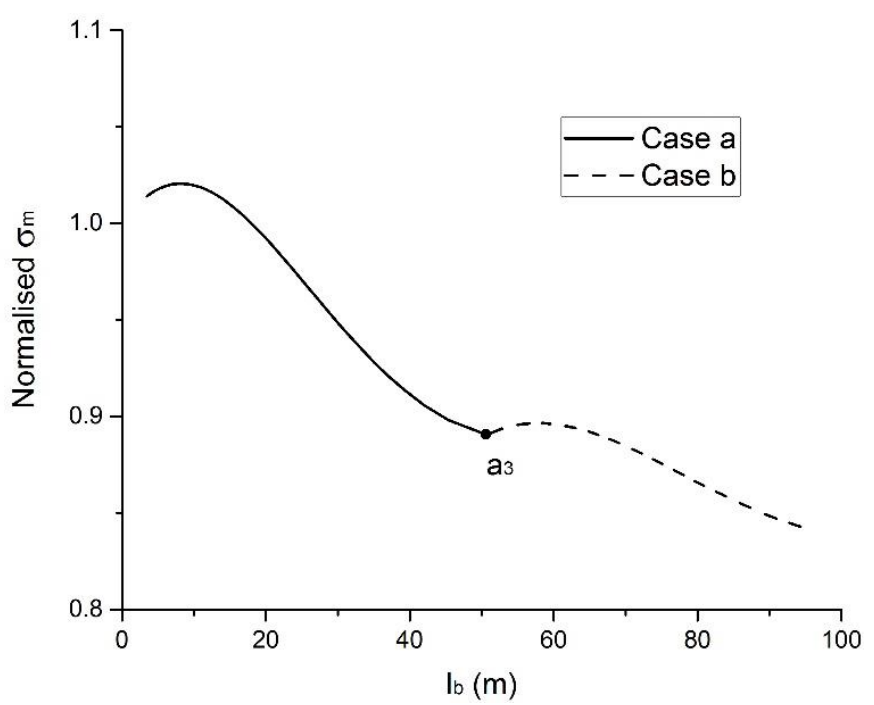

(b)

Fig. 21 Influence of $l_{b}$. (a) Normalised lateral displacement amplitude $w_{m}$. (b) Normalised maximum axial compressive stress $\sigma_{m} . k=0.6 . T_{0}=40^{\circ} \mathrm{C}$.

The influence of $l_{b}$ on the normalised lateral displacement amplitude $w_{m}$ and the normalised maximum axial compressive stress $\sigma_{m}$ for $T_{0}=40^{\circ} \mathrm{C}$ and $k=0.6$ is illustrated in Fig. 21, while the influence of $l_{b}$ on the lengths $l_{1}$ and $l_{2}$ is presented in Fig. 22. The values of $w_{\mathrm{m}}$ and $\sigma_{\mathrm{m}}$ presented in Fig. 21 are normalised against the corresponding 
values of $w_{\mathrm{m}}$ and $\sigma_{\mathrm{m}}$ for pipelines without buoyancy section. The result in Fig. 22 is used to judge whether the solution belongs to case $a$ or case $b$. In Fig. 21 and Fig. 22 a key point, namely $a_{3}$, represents the boundary of case $a$ and case $b$. In Fig. 22 (a) it is seen that for $l_{b}<l_{b}\left(a_{3}\right)$ we have $l_{b}<l_{1}$, so the solution belongs to case $a$, while for $l_{b}>l_{b}\left(a_{3}\right)$ we have $l_{1}<l_{b}<l_{2}$, so the solution belongs to case $b$. It is clear in Fig. 21 (a) that $w_{m}$ increases with increasing $l_{b}$ for case $a$, while $w_{m}$ decreases with increasing $l_{b}$ for case $b$. From Fig. 21 (b), for case $a, \sigma_{m}$ increases slightly when $l_{b}$ increases from $0 \mathrm{~m}$ to $8 \mathrm{~m}$, while $\sigma_{m}$ decreases when $l_{b}$ increases from $8 \mathrm{~m}$ to $50.64 \mathrm{~m}$. For case $b, \sigma_{m}$ also increases first and then decreases with increasing $l_{b}$.

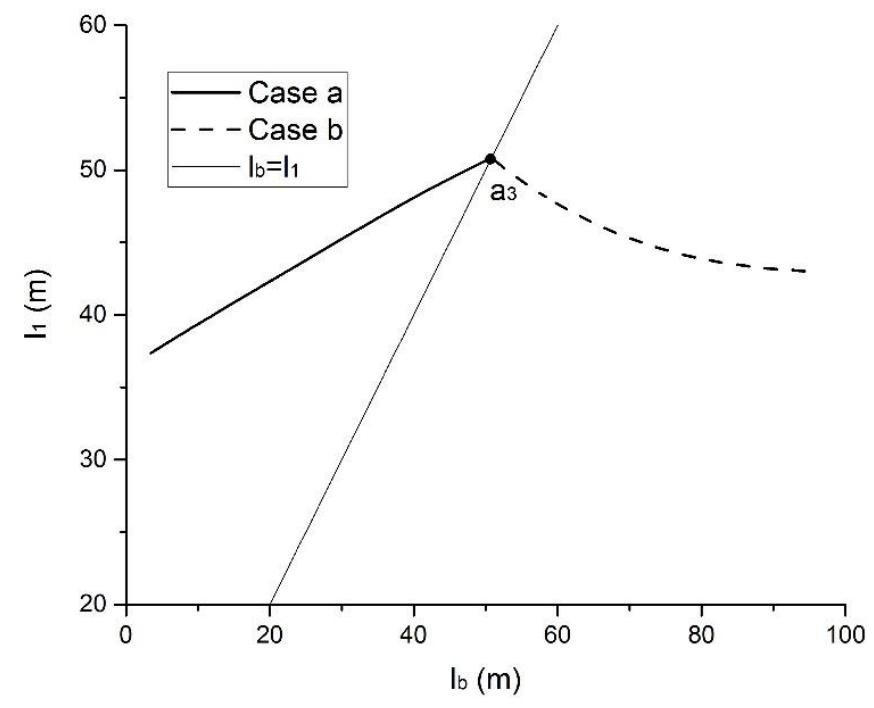

(a)

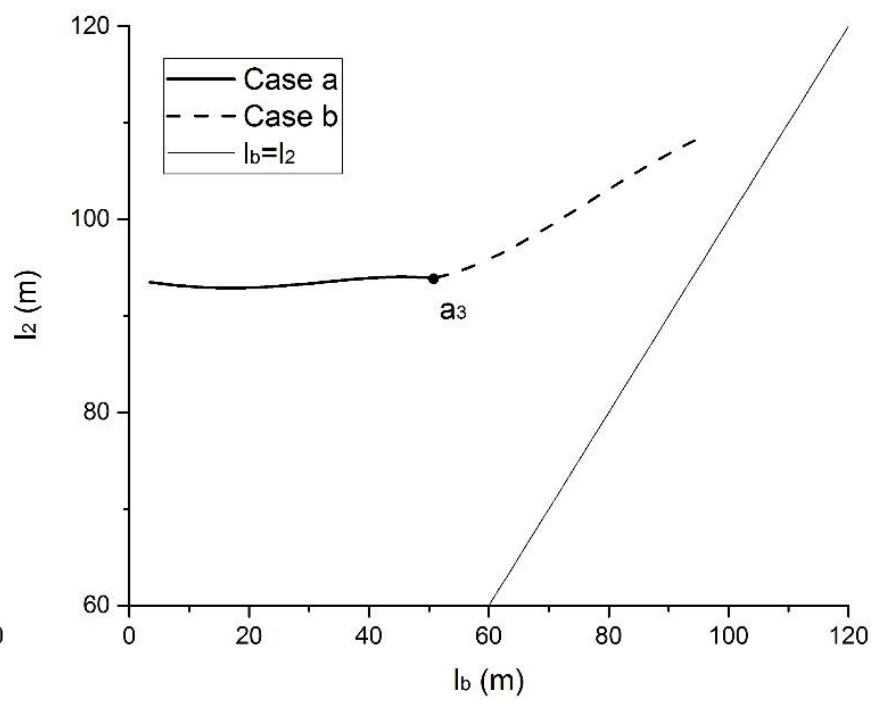

(b)

Fig. 22 Influence of $l_{b}$ on the lengths $l_{1}$ and $l_{2}$. (a) $l_{1}$. (b) $l_{2} \cdot k=0.6 . T_{0}=40{ }^{\circ} \mathrm{C}$.

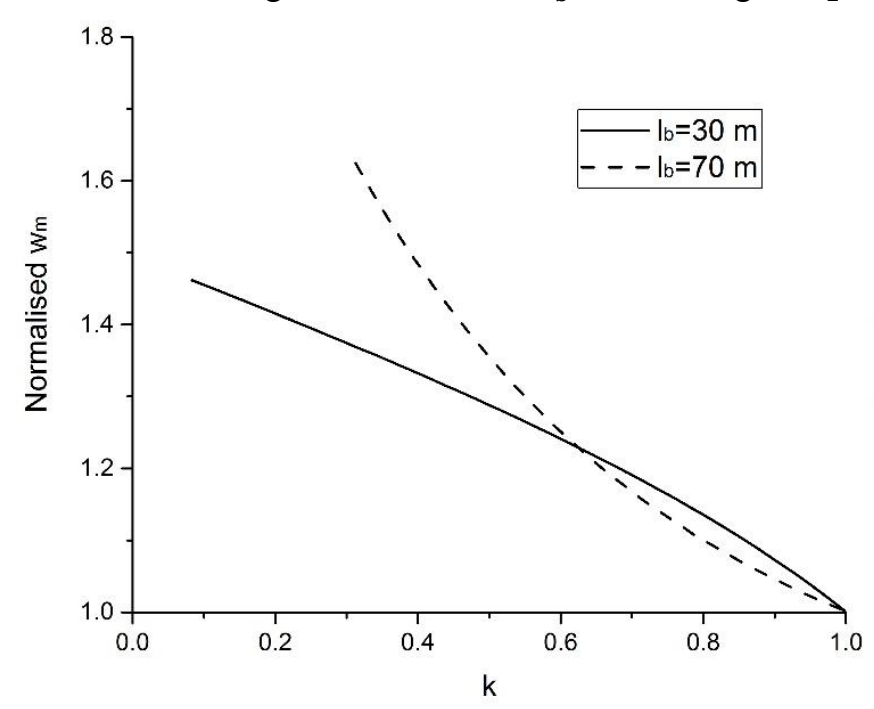

(a)

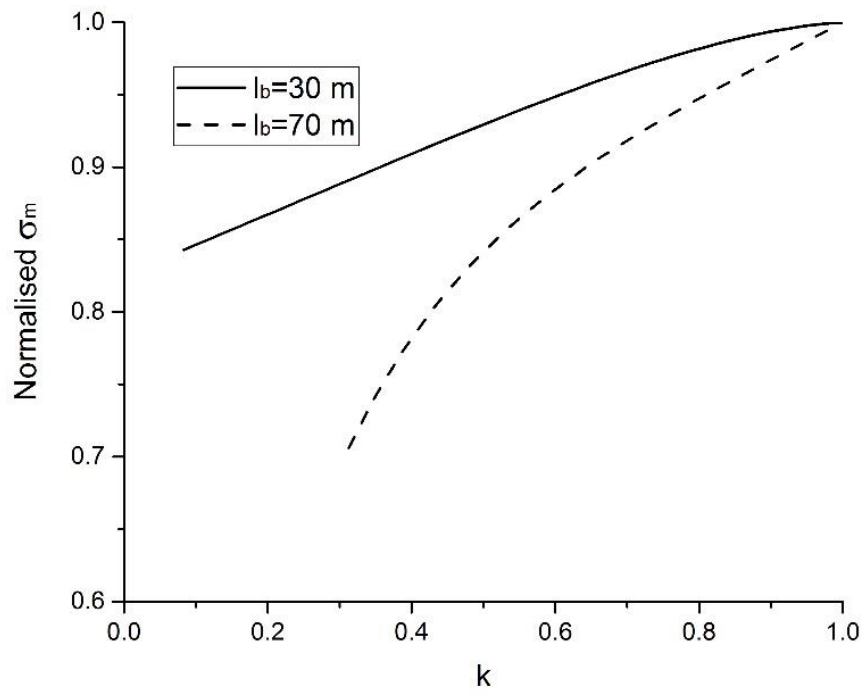

(b)

Fig. 23 Influence of $k$. (a) Normalised lateral displacement amplitude $w_{m}$. (b) Normalised maximum axial compressive stress $\sigma_{m} . T_{0}=40^{\circ} \mathrm{C}$.

The influence of $k$ on the normalised lateral displacement amplitude $w_{m}$ and the normalised maximum axial compressive stress $\sigma_{m}$ for $T_{0}=40{ }^{\circ} \mathrm{C}$ is illustrated in Fig. 23, while the influence of $k$ on the lengths $l_{1}$ and $l_{2}$ is presented in Fig. 24. The values of $w_{\mathrm{m}}$ and $\sigma_{\mathrm{m}}$ presented in Fig. 23 are normalised against the corresponding values of $w_{\mathrm{m}}$ and $\sigma_{\mathrm{m}}$ for pipelines without buoyancy section. The result in Fig. 24 is used to judge whether the solution belongs to case $a$ or case $b$. From Fig. 24 (a) and Fig. 24 (b), for $l_{b}=30 \mathrm{~m}, l_{1}>30 \mathrm{~m}$, so the solution belongs to case $a$, while for $l_{b}=$ $70 \mathrm{~m}, l_{1}<70 \mathrm{~m}<l_{2}$, so the solution belongs to case $b$. It is clear in Fig. 23 that $w_{m}$ increases with decreasing $k$, while $\sigma_{m}$ decreases with decreasing $k$, for both $l_{b}=30 \mathrm{~m}$ and $l_{b}=70 \mathrm{~m}$. The rate of increase of $w_{m}$ and the rate of decrease of $\sigma_{m}$ are almost constant with decreasing $k$ for $l_{b}=30 \mathrm{~m}$, but increase with decreasing $k$ for $l_{b}=70 \mathrm{~m}$. 


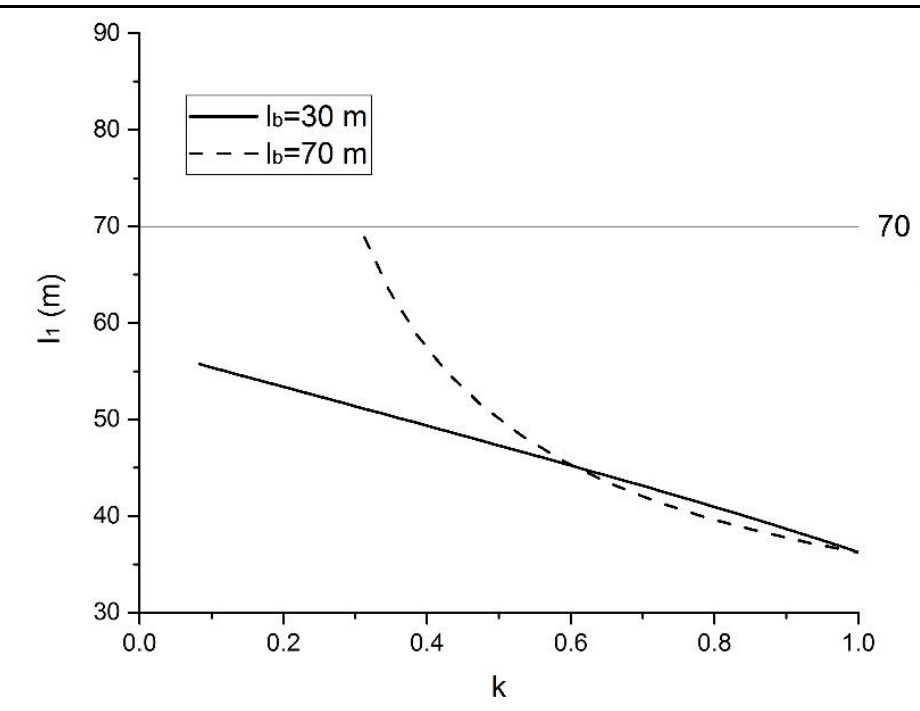

(a)

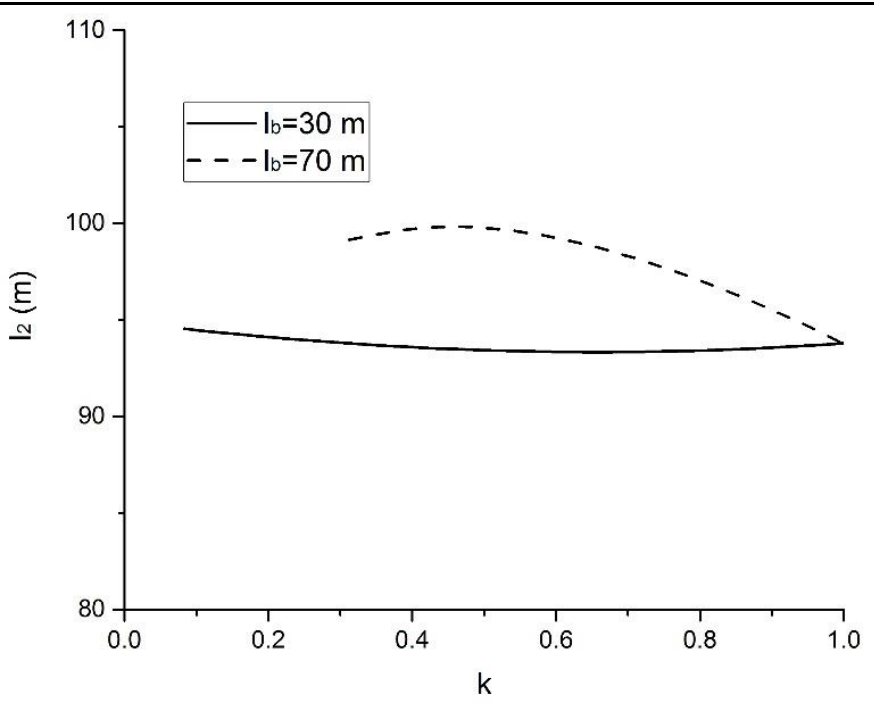

(b)

Fig. 24 Influence of $k$ on the lengths $l_{1}$ and $l_{2}$. (a) $l_{1}$. (b) $l_{2} \cdot T_{0}=40{ }^{\circ} \mathrm{C}$.

\subsection{Imperfection effect}

The analysis so far has assumed that the profile of the unbuckled pipeline is perfectly straight in the horizontal plane, namely no horizontal offset. Deviations from a straight profile for pipelines are introduced by the pipe-laying vessel's sway motion during the installation process. Large lateral offsets may be difficult to implement during installation; only very small offsets are likely during normal installation [36, 37]. The out of-straightness or initial lateral imperfection can lower the safe temperature difference and affect the post-buckling behaviour. Therefore, it is important to investigate the effect of imperfections on the lateral buckling behaviour of pipelines with distributed buoyancy section. In this analysis, a lateral deflection imperfection is imposed around the location of the buoyancy section, the configuration of which is the configuration obtained in this paper, namely $w_{1}, w_{2}$ and $w_{3}$. So only the amplitude of the initial imperfection $w_{\mathrm{om}}$ should be applied. There are two possible states for a pipeline initial imperfection: unstressed or stressed pipe. The unstressed pipe corresponds to a local imperfection in the pipe itself, which means the initial state of the pipeline with such an imperfection is unstressed. The stressed pipe represents the case where the unstressed pipeline is straight and where it forms an initial curvature due to the pipe-laying vessel's sway motion or foundation irregularities. So the imperfection included here is the stressed case. For this case, the equations governing the horizontal deflection will not be affected. The effect of the initial imperfection is that an initial geometric shortening $u_{20}$ exists. This shortening $u_{20}$ can be calculated by Eq. (29) when the amplitude of initial imperfection $w_{\text {om }}$ is given. So, Eq. (30) should be rewritten as

$$
P_{0}=P+f_{A b} l_{b}+f_{A 1}\left(-l_{b}+\sqrt{\frac{\left(f_{A 1}-f_{A b}\right) l_{b}^{2}+2 A E\left(u_{2}-u_{20}\right)}{f_{A 1}}}\right)
$$

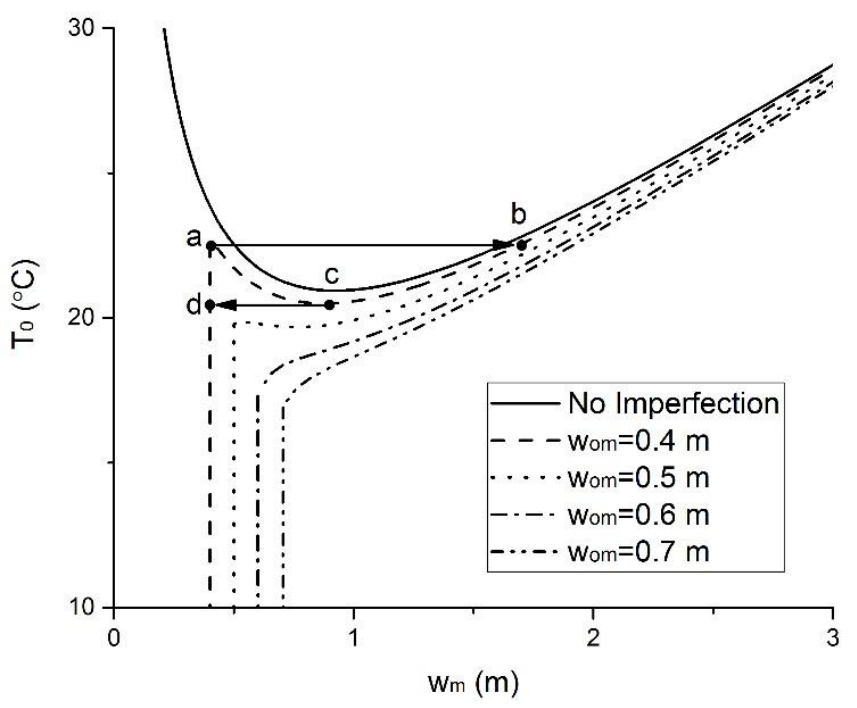


Fig. 25 The effect of imperfections on the load-deflection behavior. Arrows indicate dynamic jumps under increasing (to the right) or decreasing (to the left) $T_{0}$.

The effect of imperfections on the load-deflection behaviour of the pipeline is illustrated in Fig. 25, which shows an enlargement of the region of interest in Fig. 3 with some typical imperfection curves added. It is seen that for smaller values of the imperfection amplitude, such as $w_{\mathrm{om}}=0.4 \mathrm{~m}$, the load-deflection curves have folds where dynamic jumps of the structure may occur under both increasing and decreasing temperature. Taking $w_{\mathrm{om}}=0.4 \mathrm{~m}$ as an example, when the temperature difference increases to $T_{0}(a)$, the pipeline will jump from point $a$ to point $b$ if some disturbance occurs. With further increasing temperature difference, the pipeline will follow the post-buckling path. When the temperature difference decreases from $T_{0}(b)$ to $T_{0}(c)$, the pipeline will follow the post-buckling path from point $b$ to point $c$. Then, the pipeline will jump from point $c$ back to point $d$. For larger values of the imperfection amplitude, such as $w_{\text {om }}=0.7 \mathrm{~m}$, this snapthrough phenomenon disappears.

\subsection{Error analysis and validation}

The main approximation made in the theory is that the lateral buckling solution is calculated under the assumption of a constant compressive force $P$ (cf. Eq. (3) and Eq. (4)), an approximation generally made in the literature (e.g., [9]). In doing this the extra contributions to the pressure for $0<x<l_{2}$ in Eq. (1) are ignored. However, it is good to stress that this approximation is only made in calculating the shape of the lateral buckling solution and not in the computation of the corresponding temperature difference (based on deformational compatibility) and not in the energy analysis in Section 3.1.

In order to investigate the error incurred by this constant $P$ approximation, the lateral buckling solution with constant compressive force $P_{1}=P+f_{A b} l_{b}+f_{A 1}\left(l_{2}-l_{b}\right)$ is also calculated, and denoted by case $a-l_{2} . P_{1}$ is the actual axial compressive force at $x=l_{2}$. Since $P_{1}$ is the maximum compressive force attained in the buckled configuration, case $a-l_{2}$ will give an upper bound to the error. The lateral displacement amplitude and maximum axial compressive stress of case $a$ $l_{2}$ are denoted by $w_{m b}$ and $\sigma_{m b}$, respectively. The two cases are compared in Fig. 26 and Fig. 27. Fig. 26 shows that the difference between case $a$ and case $a-l_{2}$ is very small, the critical temperatures being $20.48{ }^{\circ} \mathrm{C}$ and $19.64{ }^{\circ} \mathrm{C}$, respectively (an error of $4.1 \%$ ). In Fig. 27 it is seen that the error of lateral displacement amplitude and maximum axial compressive stress between case $a$ and case $a-l_{2}$ is large around the critical temperature, but decreases rapidly to $-5 \%-5 \%$ away from the critical temperature. The large relative error near criticality is natural since the lateral deflection is so small. It causes no problems in practice as the absolute stress levels involved are very low (see Fig. 26).

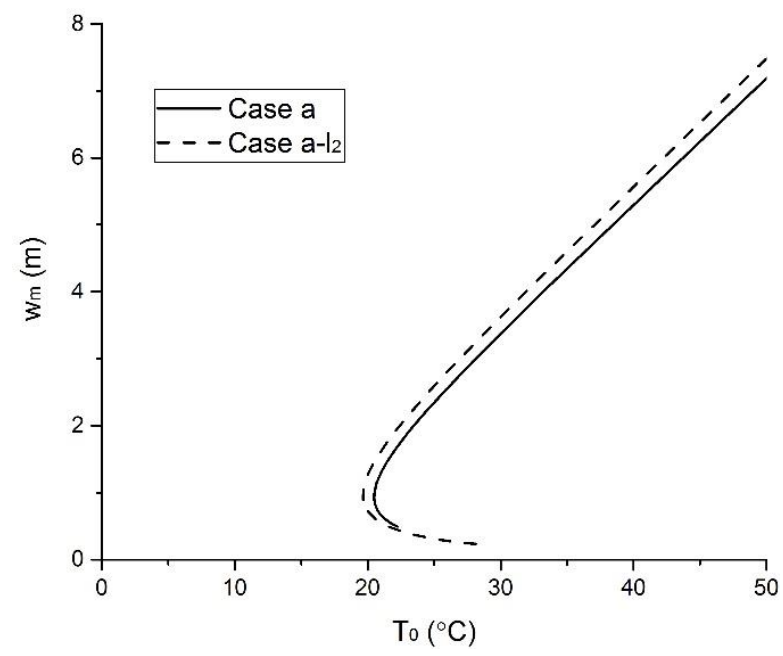

(a)

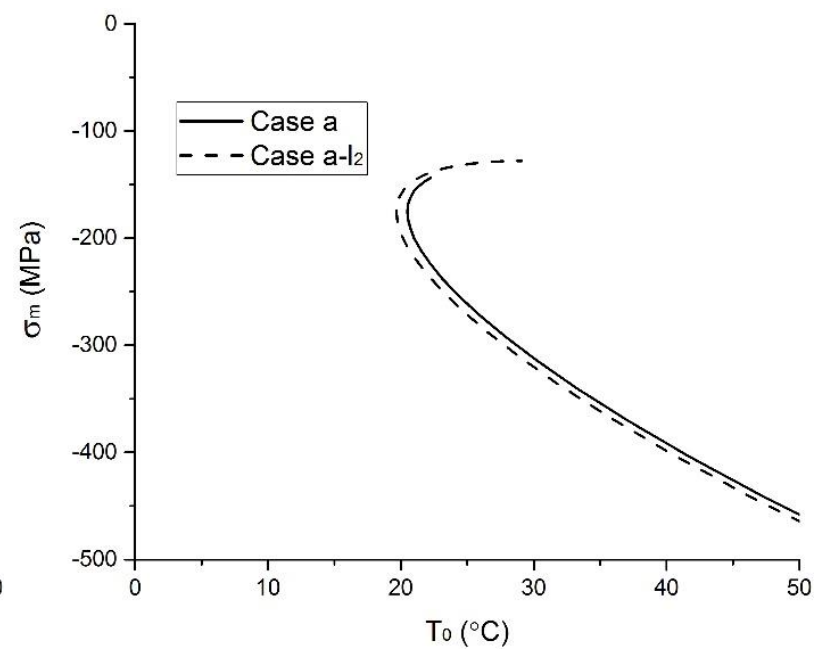

(b)

Fig. 26 Comparison of case $a$ and case $a-l_{2}$. (a) Lateral displacement amplitude. (b) Maximum axial compressive stress. $l_{b}=25 \mathrm{~m} . k=0.6$. 


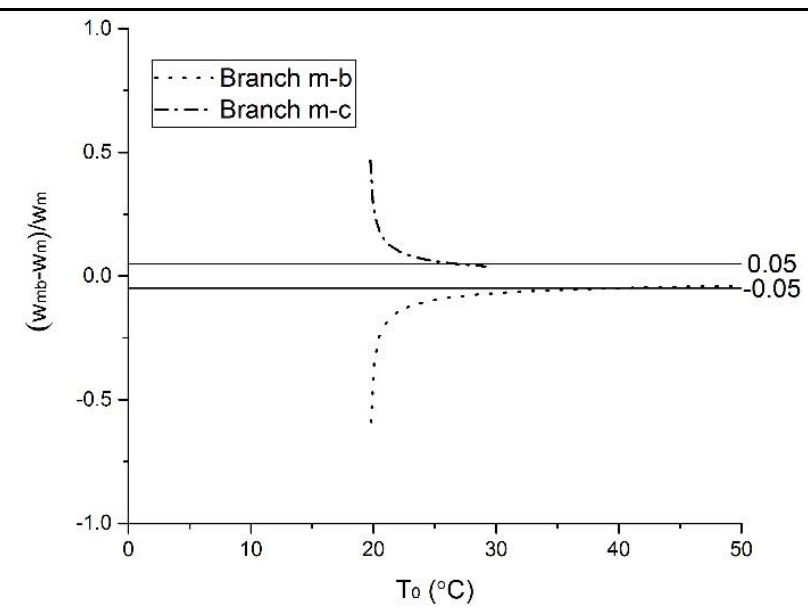

(a)

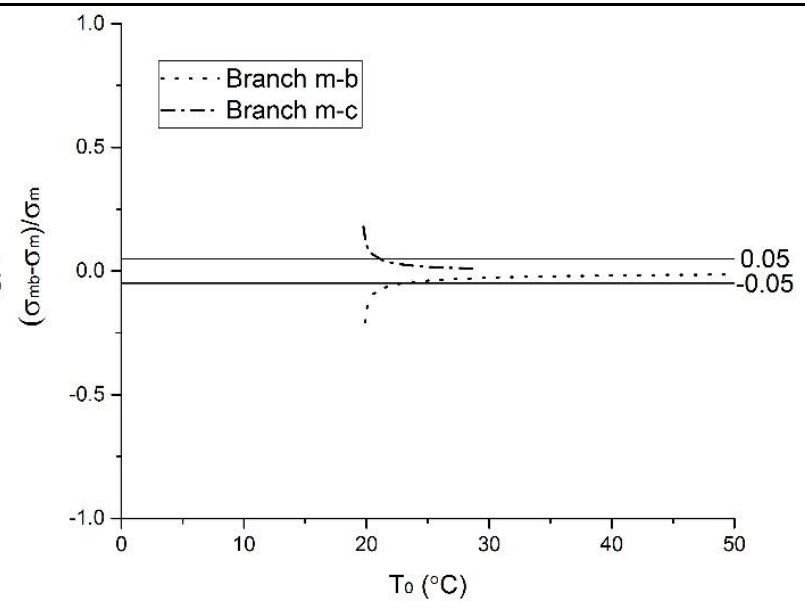

(b)

Fig. 27 Error between case $a$ and case $a-l_{2}$. (a) $\left(w_{m b}-w_{m}\right) / w_{m}$. (b) $\left(\sigma_{m b}-\sigma_{m}\right) / \sigma_{m} . l_{b}=25 \mathrm{~m} . k=0.6$.

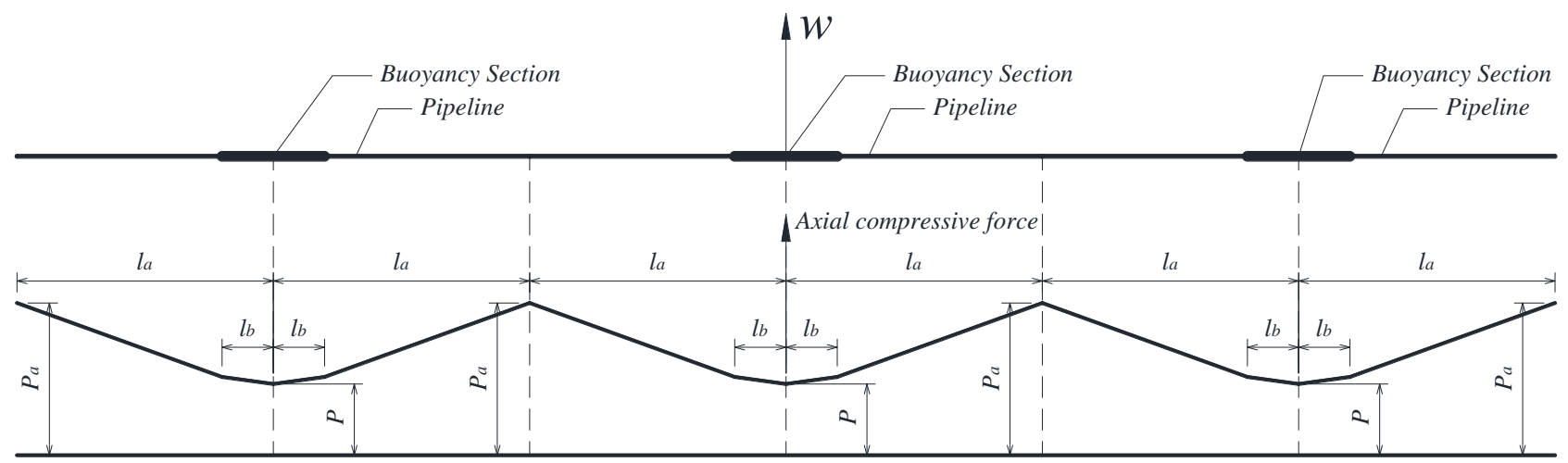

Fig. 28 Pipeline with three distributed buoyancy sections and the corresponding axial compressive force distribution.

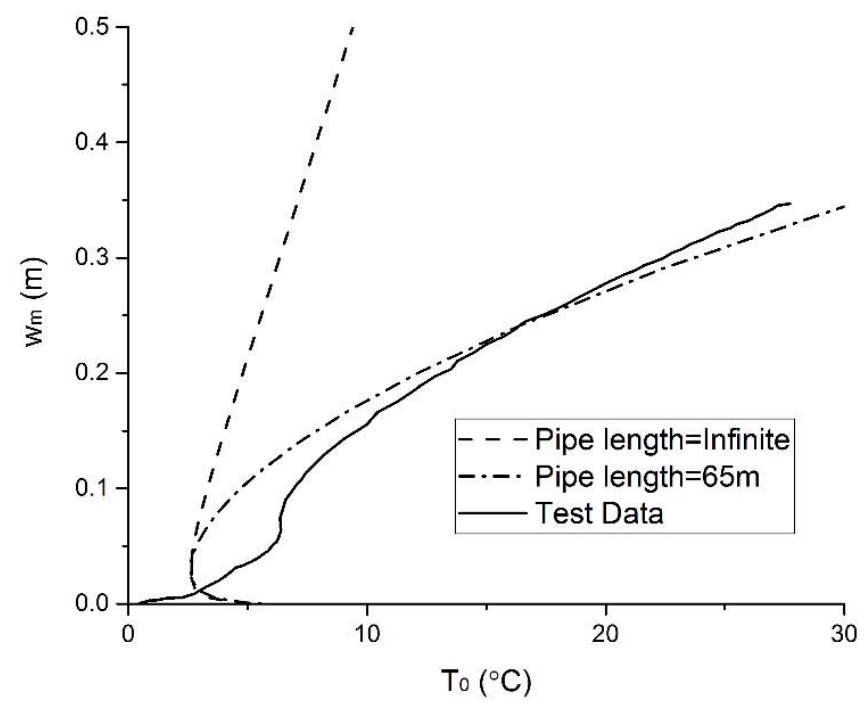

Fig. 29 Validation with test data.

To validate the analytical results proposed in this paper, another case is calculated by using the test parameters listed in Table 2. These parameters are the same as those used in the reduced-scale model in [34]. The total pipe length in [34] was $195 \mathrm{~m}$. Three distributed buoyancy sections with equal spacing of $2 l_{a}=65 \mathrm{~m}$ were installed to trigger lateral buckling, as shown in Fig. 28. $l_{a}$ represents the maximum possible axial feed-in length. $P_{a}$ is the axial compressive force at the virtual anchors between two buckles. By symmetry, the axial feed-in displacement at the midpoint between two buoyancy sections, namely at the virtual anchor between two buckles, is zero. So, for comparison with our model, a pipeline section with one 
buoyancy section, and of length $L=65 \mathrm{~m}$, is considered here. For this case, the maximum axial feed-in length $l_{a}$ is $32.5 \mathrm{~m}$. When the feed-in length $l_{s}$ is smaller than this maximum value $l_{a}$ then two adjacent buckles triggered by two adjacent buoyancy sections are independent and all the formulae derived in this paper can be applied. When $l_{s}$ is larger than $l_{a}$, the formula for axial compressive force should be modified due to the limit of axial feed-in length. In that case, by axial force balance, the following equation can be obtained [38]

$$
P_{a}=P+f_{A 1}\left(l_{a}-l_{\mathrm{b}}\right)+f_{A b} l_{\mathrm{b}}
$$

and the length of axial expansion within the pipeline section $0<x<l_{a}$ should be modified to

$$
u_{1}=\int_{0}^{l_{a}} \frac{\Delta \bar{P}(x)}{E A} d x
$$

where

$$
\Delta \bar{P}(x)= \begin{cases}P_{0}-P-f_{A b} x & 0<x<l_{\mathrm{b}} \\ P_{0}-P-f_{A b} l_{\mathrm{b}}-f_{A 1}\left(x-l_{\mathrm{b}}\right) & l_{\mathrm{b}}<x<l_{a}\end{cases}
$$

Then, given $T_{0}$ and $l_{a}$, Eq. (24), Eq. (29), Eq. (31), Eq. (43) and Eq. (44) can be solved in conjunction with Eq. (9), Eq. (10) and Eq. (11) (with Eq. (7) inserted) for case $a$, or with Eq. (9), Eq. (12) and Eq. (13) (with Eq. (8) inserted) for case $b$, to obtain $P, l_{1}, l_{2}$ and the coefficients $A_{1}-A_{12}$ for case $a$, or $B_{1}-B_{12}$ for case $b$, and hence the lateral deflections $w_{1}$, $w_{2}$ and $w_{3}$.

The results from model test and analytical method are compared in Fig. 29. The present analytical results appear to agree well with the test data for $10.5^{\circ} \mathrm{C}<T_{0}<27.5^{\circ} \mathrm{C}$. For $T_{0}=10.5^{\circ} \mathrm{C}$, the error between analytical result and test data is $7.2 \%$. For $T_{0}=27.5^{\circ} \mathrm{C}$, the error between analytical result and test data is $5.5 \%$. The errors are smaller than these two errors for $10.5^{\circ} \mathrm{C}<T_{0}<27.5^{\circ} \mathrm{C}$. For comparison, results (labelled 'Pipe length=infinite') obtained by assuming that always $l_{s}<l_{a}$, i.e., that the required feed-in length $l_{s}$ is always available, are also included in Fig. 29. It is noted that the lateral displacement amplitude $w_{m}$ for a pipe of length $65 \mathrm{~m}$ is much smaller than that for a pipe of 'infinite length'. This explains why multiple buoyancy sections should be installed at regular intervals to trigger less severe pipeline buckling at multiple planned locations.

Table 2. Test parameters

\begin{tabular}{ccc}
\hline Parameter & Value & Unit \\
\hline External diameter $D$ & 15 & $\mathrm{~mm}$ \\
Wall thickness $t$ & 0.9 & $\mathrm{~mm}$ \\
Elastic modulus $E$ & 191 & $\mathrm{GPa}$ \\
Pipeline submerged weight & 3.94 & $\mathrm{~N} / \mathrm{m}$ \\
Coefficient of thermal expansion $\alpha$ & $1.73 \times 10^{-5}$ & $/{ }^{\circ} \mathrm{C}$ \\
Lateral friction coefficient $\mu_{L}$ & 0.7 & --- \\
Axial friction coefficient $\mu_{A}$ & 0.7 & --- \\
Half-length of buoyancy section $l_{b}$ & 1.5 & $\mathrm{~m}$ \\
Weight ratio $k$ & 0 & --- \\
Pipe length $L$ & 65 & $\mathrm{~m}$ \\
\hline
\end{tabular}

\section{Discussion and Conclusions}

Analytical solutions for the lateral buckling of unburied subsea pipelines with distributed buoyancy section have been derived. The solutions are based on small-deflection (beam) theory, but take exact account of the compatibility between axial and lateral deformation to obtain curves of lateral deflection and axial compressive stress against temperature difference $T_{0}$.

From our parameter studies the following conclusions can be drawn:

(i) An energy analysis reveals several critical temperatures for pipelines with and without distributed buoyancy section (see Fig. 3). No lateral buckling solutions exist for temperatures less than the minimum critical temperature difference, which therefore represents an upper bound to safe operating temperatures for the pipeline. For temperatures larger than the minimum critical temperature difference, two solutions with distributed buoyancy section are available. Initially these have larger energy 
than the unbuckled pipe. However, typically, for only slightly higher temperatures $\left(T_{0}>T_{e}\right)$ one of the solutions acquires an energy lower than that of the trivial solution (see Fig. 4). For such temperatures, the unbuckled pipe with distributed buoyancy section can therefore be considered unstable under sufficiently large perturbations (e.g., dynamic disturbances due to irregular fluid flow through the pipe or earthquakes). Meanwhile, the pipeline without distributed buoyancy section becomes similarly unstable under large perturbations at the higher temperature $T_{\mathrm{m} 1}$ (it was shown in [24] that the trivial solution becomes eventually unstable under small perturbations in a Hamiltonian-Hopf bifurcation at much larger temperature difference). This instability, however, is sudden and may occur anywhere along the pipe. Thus, by introducing a controlled deformation at a specific location, a distributed buoyancy section causes the pipeline to buckle laterally at lower temperature difference, thereby avoiding a potentially hazardous instability.

(ii) For pipelines with distributed buoyancy section, under the same operating temperature difference, the primary lobe increases while the secondary lobe decreases with increasing length of buoyancy section for case $a$. However, for case $b$, the primary lobe decreases while the secondary lobe increases with increasing length of buoyancy section. For both case $a$ and case $b$, the primary lobe grows and the secondary lobe shrinks with decreasing weight ratio.

(iii) The minimum critical temperature difference decreases with increasing length of buoyancy section and decreasing weight ratio for both case $a$ and case $b$. However, for a given weight ratio, the rate of decrease of minimum critical temperature difference for case $a$ is much larger than that for case $b$. For a given length of buoyancy section, the rate of decrease of minimum critical temperature difference for case $a$ is smaller than that for case $b$.

(iv) Under the same operating temperature difference, for a given weight ratio, lateral displacement amplitude increases with increasing length of buoyancy section for case $a$, while lateral displacement amplitude decreases with increasing length of buoyancy section for case $b$. Also, for both case $a$ and case $b$, the maximum axial compressive stress increases slightly first and then decreases with increasing length of the buoyancy section. For a given length of the buoyancy section, the lateral displacement amplitude increases while the maximum axial compressive stress decreases with decreasing weight ratio for both case $a$ and case $b$. The rate of increase of the lateral displacement amplitude and the rate of decrease of the maximum axial compressive stress are almost constant with decreasing weight ratio for case $a$, but increase with decreasing weight ratio for case $b$.

(v) An imperfection analysis shows that a snap-through instability occurs for relatively small values of the imperfection amplitude. For sufficiently large imperfection amplitude this snap-through instability disappears.

(vi) An error analysis shows that the assumption of constant axial compressive force for the calculation of lateral deflection is acceptable. A comparison between the analytical solution and test data shows good agreement.

(vii) In this paper the case of a single buoyancy section is considered, but in Section 3.5 it is indicated how the model can be extended to multiple buoyancy sections, which may be employed to significantly reduce lateral buckling amplitudes.

\section{Acknowledgments}

The authors would like to acknowledge that the work described in this paper was funded by the National Key Basic Research Program of China (2014CB046805).

\section{Appendix A.}

$$
\begin{aligned}
A_{1} & =\frac{\sec \left(\lambda l_{2}\right)\left(\left(f_{1}-f_{\mathrm{b}}\right) \cos \left(\lambda\left(l_{2}-l_{\mathrm{b}}\right)\right)-2 f_{1} \cos \left(\lambda\left(l_{1}-l_{2}\right)\right)+f_{1}\right)}{\operatorname{EI} \lambda^{4}} \\
A_{2} & =0 \\
A_{3} & =0 \\
A_{4} & =\frac{2 f_{\mathrm{b}}-\lambda^{2}\left(f_{1}\left(2 l_{1}^{2}-4 l_{1} l_{2}+l_{2}^{2}+2 l_{2} l_{\mathrm{b}}-l_{\mathrm{b}}^{2}\right)+f_{\mathrm{b}} l_{\mathrm{b}}\left(l_{\mathrm{b}}-2 l_{2}\right)\right)}{2 \mathrm{E} \lambda^{4}} \\
A_{5} & =\frac{\left(f_{1}-f_{\mathrm{b}}\right) \tan \left(\lambda l_{2}\right) \sin \left(\lambda l_{\mathrm{b}}\right)+\sec \left(\lambda l_{2}\right)\left(f_{1}-2 f_{1} \cos \left(\lambda\left(l_{1}-l_{2}\right)\right)\right)}{\operatorname{EI} \lambda^{4}}
\end{aligned}
$$




$$
\begin{aligned}
& A_{6}=\frac{\left(f_{\mathrm{b}}-f_{1}\right) \sin \left(\lambda l_{\mathrm{b}}\right)}{\operatorname{EI} \lambda^{4}} \\
& A_{7}=\frac{l_{\mathrm{b}}\left(f_{1}-f_{\mathrm{b}}\right)}{\mathrm{EI}^{2}} \\
& A_{8}=\frac{f_{1}\left(2-\lambda^{2}\left(2 l_{1}^{2}-4 l_{1} l_{2}+l_{2}\left(l_{2}+2 l_{\mathrm{b}}\right)\right)\right)+2 f_{\mathrm{b}} \lambda^{2} l_{2} l_{\mathrm{b}}}{2 \mathrm{EI} \lambda^{4}} \\
& A_{9}=\frac{\tan \left(\lambda l_{2}\right)\left(\left(f_{1}-f_{\mathrm{b}}\right) \sin \left(\lambda l_{\mathrm{b}}\right)-2 f_{1} \sin \left(\lambda l_{1}\right)\right)+f_{1} \sec \left(\lambda l_{2}\right)}{\operatorname{EI} \lambda^{4}} \\
& A_{10}=\frac{\left(f_{\mathrm{b}}-f_{1}\right) \sin \left(\lambda l_{\mathrm{b}}\right)+2 f_{1} \sin \left(\lambda l_{1}\right)}{\operatorname{EI} \lambda^{4}} \\
& A_{11}=\frac{f_{1}\left(l_{\mathrm{b}}-2 l_{1}\right)-f_{\mathrm{b}} l_{\mathrm{b}}}{\mathrm{EI} \lambda^{2}} \\
& A_{12}=\frac{\lambda^{2} l_{2}\left(f_{1}\left(4 l_{1}-l_{2}-2 l_{\mathrm{b}}\right)+2 f_{\mathrm{b}} l_{\mathrm{b}}\right)-2 f_{1}}{2 \mathrm{EI} \lambda^{4}} \\
& B_{1}=\frac{\sec \left(\lambda l_{2}\right)\left(\left(f_{\mathrm{b}}-f_{1}\right) \cos \left(\lambda\left(l_{2}-l_{\mathrm{b}}\right)\right)+f_{1}-2 f_{\mathrm{b}} \cos \left(\lambda\left(l_{1}-l_{2}\right)\right)\right)}{E I \lambda^{4}} \\
& B_{2}=0 \\
& B_{3}=0 \\
& B_{4}=\frac{f_{\mathrm{b}}\left(\lambda^{2}\left(-2 l_{1}^{2}+4 l_{1} l_{2}+l_{\mathrm{b}}\left(l_{\mathrm{b}}-2 l_{2}\right)\right)+2\right)-f_{1} \lambda^{2}\left(l_{2}-l_{\mathrm{b}}\right)^{2}}{2 E I \lambda^{4}} \\
& B_{5}=\frac{\tan \left(\lambda l_{2}\right)\left(\left(f_{\mathrm{b}}-f_{1}\right) \sin \left(\lambda l_{\mathrm{b}}\right)-2 f_{\mathrm{b}} \sin \left(\lambda l_{1}\right)\right)+\left(f_{\mathrm{b}}-f_{1}\right) \cos \left(\lambda l_{\mathrm{b}}\right)+f_{1} \sec \left(\lambda l_{2}\right)}{\operatorname{EI} \lambda^{4}} \\
& B_{6}=\frac{2 f_{\mathrm{b}} \sin \left(\lambda l_{1}\right)}{E I \lambda^{4}} \\
& B_{7}=-\frac{2 f_{\mathrm{b}} l_{1}}{E I \lambda^{2}} \\
& B_{8}=\frac{\lambda^{2}\left(f_{\mathrm{b}}\left(4 l_{1} l_{2}+l_{\mathrm{b}}\left(l_{\mathrm{b}}-2 l_{2}\right)\right)-f_{1}\left(l_{2}-l_{\mathrm{b}}\right)^{2}\right)-2 f_{\mathrm{b}}}{2 E I \lambda^{4}} \\
& B_{9}=\frac{\tan \left(\lambda l_{2}\right)\left(\left(f_{\mathrm{b}}-f_{1}\right) \sin \left(\lambda l_{\mathrm{b}}\right)-2 f_{\mathrm{b}} \sin \left(\lambda l_{1}\right)\right)+f_{1} \sec \left(\lambda l_{2}\right)}{E I \lambda^{4}} \\
& B_{10}=\frac{\left(f_{1}-f_{\mathrm{b}}\right) \sin \left(\lambda l_{\mathrm{b}}\right)+2 f_{\mathrm{b}} \sin \left(\lambda l_{1}\right)}{E I \lambda^{4}} \\
& B_{11}=\frac{f_{\mathrm{b}}\left(l_{\mathrm{b}}-2 l_{1}\right)-f_{1} l_{\mathrm{b}}}{E I \lambda^{2}} \\
& B_{12}=\frac{\lambda^{2} l_{2}\left(-f_{1} l_{2}+2 f_{1} l_{\mathrm{b}}+4 f_{\mathrm{b}} l_{1}-2 f_{\mathrm{b}} l_{\mathrm{b}}\right)-2 f_{1}}{2 E I \lambda^{4}}
\end{aligned}
$$

\section{References}

[1] Bruton D. The safe design of hot on bottom pipelines with lateral buckling using the design-guideline developed by the SAFEBUCK joint industry project. Deep Offshore Technology Conference, Vitoria, Espirito Santo, Brazil, 2005.

[2] White DJ, Cheuk CY. Modelling the soil resistance on seabed pipelines during large cycles of lateral movement. Marine Structures. 2008;21:59-79.

[3] Wang Z, Tang Y, Feng H, Zhao Z, Liu H. Model test for lateral soil resistance of partially embedded subsea pipelines on sand during large-amplitude lateral movement. J Coast Res. 2017;333:607-18.

[4] Shi R, Wang L. Single buoyancy load to trigger lateral buckles in pipelines on a soft seabed. Journal of Engineering Mechanics. 
2015;141:1-7.

[5] Reddy R. Lateral buckling behaviour of snake-lay pipeline with vertical support at crown. ASME $32^{\text {nd }}$ International Conference on Ocean, Offshore and Arctic Engineering, Nantes, France, 2013.

[6] Sinclair F, Carr M, Bruton D, Farrant T. Design challenges and experience with controlled lateral buckle initiation methods. ASME $28^{\text {th }}$ International Conference on Ocean, Offshore and Arctic Engineering, Honolulu, Hawaii, USA, 2009.

[7] Urthaler Y, Watson R, Davis J. Lateral buckling of deepwater pipelines in operation. ASME $31^{\text {st }}$ International Conference on Ocean, Offshore and Arctic Engineering, Rio de Janeiro, Brazil, 2012.

[8] Hobbs RE. Pipeline buckling caused by axial loads. Journal of Constructional Steel Research. 1981;1:2-10.

[9] Hobbs RE. In-service buckling of heated pipelines. Journal of Transportation Engineering. 1984;110:175-89.

[10] Taylor N, Gan AB. Submarine pipeline buckling-imperfection studies. Thin-Walled Structures. 1986;4:295-323.

[11] Taylor N, Tran V. Experimental and theoretical studies in subsea pipeline buckling. Marine Structures. 1996;9:211-57.

[12] Taylor N, Tran V. Prop-imperfection subsea pipeline buckling. Marine Structures. 1993;6:325-58.

[13] Taylor N, Gan AB. Refined modelling for the vertical buckling of submarine pipelines. Journal of Constructional Steel Research. 1987;7:55-74.

[14] Taylor N, Gan AB. Refined modelling for the lateral buckling of submarine pipelines. Journal of Constructional Steel Research. 1986;6:143-62.

[15] Pedersen PT, Jensen JJ. Upheaval creep of buried heated pipelines with initial imperfections. Marine Structures. 1988;1:1122.

[16] Wang L, Shi R, Yuan F, Guo Z, Yu L. Global buckling of pipelines in the vertical plane with a soft seabed. Applied Ocean Research. 2011;33:130-6.

[17] Shi R, Wang L, Guo Z, Yuan F. Upheaval buckling of a pipeline with prop imperfection on a plastic soft seabed. Thin-Walled Structures. 2013;65:1-6.

[18] Hong Z, Liu R, Liu W, Yan S. Study on lateral buckling characteristics of a submarine pipeline with a single arch symmetric initial imperfection. Ocean Eng. 2015;108:21-32.

[19] Liu R, Liu W, Wu X, Yan S. Global lateral buckling analysis of idealized subsea pipelines. Journal of Central South University. 2014;21:416-27.

[20] Karampour H, Albermani F, Gross J. On lateral and upheaval buckling of subsea pipelines. Engineering Structures. 2013;52:317-30.

[21] Karampour H, Albermani F. Experimental and numerical investigations of buckle interaction in subsea pipelines. Engineering Structures. 2014;66:81-8.

[22] Karampour H, Albermani F, Veidt M. Buckle interaction in deep subsea pipelines. Thin-Walled Structures. 2013;72:113-20.

[23] Zhu J, Attard MM, Kellermann DC. In-plane nonlinear localised lateral buckling of straight pipelines. Engineering Structures. 2015;103:37-52.

[24] Wang Z, van der Heijden GHM. Localised lateral buckling of partially embedded subsea pipelines with nonlinear soil resistance. Thin-Walled Structures. 2017;120:408-20.

[25] Wang Z, Chen Z, Liu H, Bu Y. Static and dynamic analysis on upheaval buckling of unburied subsea pipelines. Ocean Eng. 2015;104:249-56.

[26] Wang Z, Chen Z, Liu H. Numerical study on upheaval buckling of pipe-in-pipe systems with full contact imperfections. Engineering Structures. 2015;99:264-71.

[27] Liu R, Basu P, Xiong H. Laboratory tests and thermal buckling analysis for pipes buried in Bohai soft clay. Marine Structures. 2015;43:44-60.

[28] Hong Z, Liu R, Liu W, Yan S. A lateral global buckling failure envelope for a high temperature and high pressure (HT/HP) submarine pipeline. Applied Ocean Research. 2015;51:117-28.

[29] Zhang X, Duan M. Prediction of the upheaval buckling critical force for imperfect submarine pipelines. Ocean Eng. 2015;109:330-43.

[30] Peek R, Yun H. Flotation to trigger lateral buckles in pipelines on a flat seabed. Journal of Engineering Mechanics. 
2007;4:442-51.

[31] Antunes BR, Solano RF, Vaz MA. Analytical formulation of distributed buoyancy sections to control lateral buckling of subsea pipelines. ASME $29^{\text {th }}$ International Conference on Ocean, Offshore and Arctic Engineering, Shanghai, China, 2010.

[32] Li G, Zhan L, Li H. An analytical solution to lateral buckling control of subsea pipelines by distributed buoyancy sections. Thin-Walled Structures. 2016;107:221-30.

[33] Wang Z, Tang Y, Wang C. Analytical solution for lateral buckling of unburied subsea pipelines with distributed buoyancy section. Ocean Eng. 2017;146:115-24.

[34] de Oliveira Cardoso C, Solano RF. Performed of triggers to control thermal buckling of subsea pipelines using reduced scale model. $25^{\text {th }}$ International Ocean and Polar Engineering Conference, International Society of Offshore and Polar Engineers, Kona, Hawaii, 2015.

[35] DNV-RP-F110. Global buckling of submarine pipelines structural design due to high temperature/high pressure. Det Norske Veritas; 2007.

[36] Bai Q, Qi X, Brunner M. Global buckle control with dual sleepers in HP/HT pipelines. OTC-19888-MS, Offshore Technology Conference, Houston, Texas, USA, 2009.

[37] Zeng X, Duan M, Che X. Critical upheaval buckling forces of imperfect pipelines. Applied Ocean Research. 2014;45:33-9.

[38] Wang Z, Tang Y, Zhou L, Zhao Z, Wang C. Analytical solution for controlled lateral buckling of unburied subsea pipelines. Ocean Eng. 2017;146:140-50. 